International Journal of Pure and Applied Mathematics

Volume 85 No. 2 2013, 303-338

ISSN: 1311-8080 (printed version); ISSN: 1314-3395 (on-line version)

url: http://www.ijpam.eu

doi: http://dx.doi.org/10.12732/ijpam.v85i2.10

ijpam.eu

\title{
AUTONOMOUS EVOLUTIONARY INCLUSIONS WITH APPLICATIONS TO PROBLEMS WITH NONLINEAR BOUNDARY CONDITIONS
}

\author{
Sascha Trostorff \\ Institute for Analysis \\ Faculty of Mathematics and Sciences \\ Technical University Dresden \\ Dresden, GERMANY
}

\begin{abstract}
We study an abstract class of autonomous differential inclusions in Hilbert spaces and show the well-posedness and causality, by establishing the operators involved as maximal monotone operators in time and space. Then the proof of the well-posedness relies on a well-known perturbation result for maximal monotone operators. Moreover, we show that certain types of nonlinear boundary value problems are covered by this class of inclusions and we derive necessary conditions on the operators on the boundary in order to apply the solution theory. We exemplify our findings by two examples.
\end{abstract}

AMS Subject Classification: 34G25, 35F30, 35R20, 46N20, 47J35

Key Words: evolutionary inclusions, well-posedness, causality, maximal monotonicity, impedance type boundary conditions, frictional boundary conditions

\section{Introduction}

In this article we study differential inclusions of the form

$$
(u, f) \in \partial_{0} M\left(\partial_{0}^{-1}\right)+A,
$$

where $\partial_{0}$ denotes the derivative with respect to time and $A$ is assumed to be

Received: December 11, 2012

(c) 2013 Academic Publications, Ltd. url: www.acadpubl.eu 
a maximal monotone relation in time and space. The bounded linear operator $M\left(\partial_{0}^{-1}\right)$, also acting in time and space, is a so-called linear material law, as it was introduced in [15]. Since we are dealing with the autonomous case, the operator $M\left(\partial_{0}^{-1}\right)$ and the relation $A$ are assumed to commute with the temporal translation operator. We will show that under suitable conditions on $M\left(\partial_{0}^{-1}\right)$ and $A$ the problem 1 is well-posed, i.e. Hadamard's requirements on existence and uniqueness of a solution $u$ and its continuous dependence on the given data $f$ are satisfied. Moreover the issue of causality is addressed, meaning that the behaviour of the solution up to a certain time $T$ just depends on the behaviour of the given right hand side up to the same time $T$.

Originally this class of problems was discussed in [15], where the relation $A$ was given by a skew-selfadjoint spatial operator. There, well-posedness and causality were shown under a positive-definiteness constraint on the operator $M\left(\partial_{0}^{-1}\right)$. Moreover, the abstract solution theory was applied to several examples of linear equations in mathematical physics. This solution theory was generalized by the author to the case of $A$ being a maximal monotone relation in space in [27], where $M\left(\partial_{0}^{-1}\right)$ was of the particular form $M_{0}+\partial_{0}^{-1} M_{1}$. We will adopt the proof for the solution theory presented in [27] to show the well-posedness of problems of the form 1 . The proof mainly relies on the realization of the derivative $\partial_{0}$ as a maximal monotone operator on an exponentially weighted $L_{2}$-space (see e.g. [18],[21] or Subsection 2.1 of this article) and the application of a well-known perturbation result for maximal monotone relations (see 2.4). For the theory of maximal monotone relations we refer to the monographs [1],[13],[24],[5] and [6]. Inclusions of the form 1 cover a large class of possible evolutionary problems, such as integro-differential equations [28], delay and neutral differential equations [7], certain types of control problems [20],[19] and equations of mathematical physics involving hysteresis [27].

The article is structured as follows. In Section 2 we recall the definition of the time-derivative, of linear material laws and some basic facts on maximal monotone relations on Hilbert spaces. Moreover, we recall the notion of so-called abstract boundary data spaces (see [20]), which will enable us to formulate evolutionary equations with nonlinear boundary condition as an inclusion of the form 1. In Section 3 the solution theory for 1 is presented. In the remaining part of the article we consider a certain type of partial differential equations with nonlinear boundary conditions. Those problems occur for instance in frictional contact problems (see e.g. [4],[25],[11] and [10]) in the field of elasticity, where the boundary condition is given by a differential inclusion. In Subsection 4.1 we study an abstract nonlinear differential operator $A$, which is given by the restriction of a linear operator to elements, which satisfy the 
nonlinear boundary condition. We show that under certain constraints on the relation, which occurs in the boundary condition, the operator $A$ gets maximal monotone and thus, the solution theory developed in Section 3 applies. We illustrate our results in Subsection 4.2. There, in a first example we consider the wave equation with an impedance-type boundary condition. This problem was originally considered in [17] and we show that it fits in our framework. The second example deals with the equations of visco-elasticity with a frictional boundary condition, which is modelled by a differential inclusion on the boundary. A similar kind of this problem was considered in [9] for a cylindrical domain and antiplane shear deformations. Again we show that the equations are covered by our abstract inclusion 1 .

Throughout every Hilbert space is assumed to be complex. Its inner product and its norm are denoted by $\langle\cdot \mid \cdot\rangle$ and $|\cdot|$, respectively, where the inner product is assumed to be linear with respect to the second and conjugate linear with respect to the first argument. Moreover, for a Hilbert space $H$ and a closed subspace $V \subseteq H$ we denote by $\pi_{V}: H \rightarrow V$ the orthogonal projection onto $V{ }^{1}$ Then $P_{V}:=\pi_{V}^{*} \pi_{V}: H \rightarrow H$ is the orthogonal projector on $V$.

\section{Preliminaries}

\subsection{The Time Derivative and Linear Material Laws}

In this subsection we recall, how to establish the time-derivative as a normal, boundedly invertible linear operator and we recall the notion of linear material laws. For the proofs and more details we refer to [15],[7] and [18]. Let $H$ be a Hilbert space. For $\nu>0$ we define $H_{\nu, 0}(\mathbb{R} ; H)$ as the space of measurable, $H$-valued functions on $\mathbb{R}$, which are square-integrable ${ }^{2}$ with respect to the exponentially weighted Lebesgue-measure $\mu_{\nu}:=\exp (-2 \nu \cdot) \lambda$, where by $\lambda$ we denote the one-dimensional Lebesgue measure. Moreover, we set $H_{\nu, 0}(\mathbb{R}):=H_{\nu, 0}(\mathbb{R} ; \mathbb{C})$. We define the operator $\partial_{0, \nu}$ as the closure of the mapping

$$
\begin{aligned}
\left.\partial_{0, \nu}\right|_{C_{c}^{\infty}(\mathbb{R})}: C_{c}^{\infty}(\mathbb{R}) \subseteq H_{\nu, 0}(\mathbb{R}) & \rightarrow H_{\nu, 0}(\mathbb{R}) \\
\phi & \mapsto \phi^{\prime}
\end{aligned}
$$

\footnotetext{
${ }^{1}$ Note that then the adjoint $\pi_{V}^{*}: V \rightarrow H$ is just the canonical embedding.

${ }^{2}$ Throughout we identify the equivalence classes with respect to equality almost everywhere with their representatives.
} 
where by $C_{c}^{\infty}(\mathbb{R})$ we denote the space of arbitrarily differentiable functions with compact support in $\mathbb{R}$. Indeed, the operator $\partial_{0, \nu}$ turns out to be normal and continuously invertible and its inverse is given by

$$
\left(\partial_{0, \nu}^{-1} u\right)(t):=\int_{-\infty}^{t} u(s) \mathrm{d} s \quad(t \in \mathbb{R})
$$

for each $u \in H_{\nu, 0}(\mathbb{R})$. Moreover $\mathrm{R}$ e $\partial_{0, \nu}=\nu$, which gives $\left\|\partial_{0, \nu}^{-1}\right\| \leq \frac{1}{\nu}$. Indeed, one can show that the operator norm of $\partial_{0, \nu}^{-1}$ is equal to $\nu^{-1}$ (see e.g. [7]). Moreover, equation 2 shows the causality ${ }^{3}$ of $\partial_{0, \nu}^{-1}$.

Furthermore, we can give a spectral representation for $\partial_{0, \nu}$ in terms of the so-called Fourier-Laplace-transform $\mathcal{L}_{\nu}: H_{\nu, 0}(\mathbb{R}) \rightarrow L_{2}(\mathbb{R})$, which is given by $\mathcal{L}_{\nu}:=\mathcal{F} \exp (-\nu m)$, where $\mathcal{F}$ denotes the Fourier-transform in $L_{2}(\mathbb{R})$ given by the unitary extension of

$$
(\mathcal{F} \phi)(x):=\frac{1}{\sqrt{2 \pi}} \int_{\mathbb{R}} \phi(y) \exp (-\mathrm{i} x y) \mathrm{d} y \quad\left(x \in \mathbb{R}, \phi \in C_{c}^{\infty}(\mathbb{R})\right)
$$

and $\exp (-\nu m): H_{\nu, 0}(\mathbb{R}) \rightarrow L_{2}(\mathbb{R})$ is defined by

$$
(\exp (-\nu m) u)(t)=\exp (-\nu t) u(t) .
$$

Clearly, $\exp (-\nu m)$ is a unitary operator and so is $\mathcal{L}_{\nu}$. Using the well-known spectral representation of the weak derivative on $L_{2}(\mathbb{R})$ via the Fourier-transform $\mathcal{F}$, we get that

$$
\partial_{\nu, 0}=\mathcal{L}_{\nu}^{*}(\mathrm{i} m+\nu) \mathcal{L}_{\nu},
$$

where $m: \mathcal{D}(m) \subseteq L_{2}(\mathbb{R}) \rightarrow L_{2}(\mathbb{R})$ denotes the multiplication-by-the-argument operator with maximal domain, i.e. $(m f)(t):=t f(t)$ for all $f \in \mathcal{D}(m)=\{g \in$ $\left.L_{2}(\mathbb{R}) \mid(x \mapsto x g(x)) \in L_{2}(\mathbb{R})\right\}$ and $t \in \mathbb{R}$. Note that the operators $\partial_{0, \nu}$ and $\mathcal{L}_{\nu}$ can be extended to $H_{\nu, 0}(\mathbb{R} ; H) \cong H_{\nu, 0}(\mathbb{R}) \otimes H$ in the canonical way.

Next we consider a bounded strongly measurable mapping

$$
M: B_{\mathbb{C}}\left(\frac{1}{2 \nu}, \frac{1}{2 \nu}\right) \rightarrow L(H),
$$

\footnotetext{
${ }^{3}$ Let $E, F$ be vector spaces and $G: \mathcal{D}(G) \subseteq E^{\mathrm{R}} \rightarrow F^{\mathrm{R}}$. $G$ is called causal (cf. [8]), if for each $a \in \mathbb{R}$ and $f, g \in \mathcal{D}(G)$ the implication

$$
\left(\chi_{(-\infty, a]}(m)(f-g)=0\right) \Rightarrow\left(\chi_{(-\infty, a]}(m)(G(f)-G(g))=0\right)
$$

holds. By $\chi_{(-\infty, a]}(m)$ we denote the multiplication operator with the cut-off function $\chi_{(-\infty, a]}$, i.e. $\left(\chi_{(-\infty, a]}(m) f\right)(t):=\chi_{(-\infty, a]}(t) f(t)$.
} 
where $B_{\mathbb{C}}(x, r)$ denotes the open ball in $\mathbb{C}$ around the center $x \in \mathbb{C}$ with radius $r>0$. We define the bounded linear operator $M\left(\frac{1}{\mathrm{i} m+\nu}\right): L_{2}(\mathbb{R} ; H) \rightarrow$ $L_{2}(\mathbb{R} ; H)$ by setting

$$
\left(M\left(\frac{1}{\mathrm{i} m+\nu}\right) u\right)(t):=M\left(\frac{1}{\mathrm{i} t+\nu}\right) u(t) \quad(t \in \mathbb{R}) .
$$

The spectral representation 3 of the derivative $\partial_{0, \nu}$ now leads to the definition of the linear and bounded operator $M\left(\partial_{0, \nu}^{-1}\right):=\mathcal{L}_{\nu}^{*} M\left(\frac{1}{\mathrm{i} m+\nu}\right) \mathcal{L}_{\nu}: H_{\nu, 0}(\mathbb{R} ; H) \rightarrow$ $H_{\nu, 0}(\mathbb{R} ; H)$. If we assume additionally that $M$ is analytic, we call $M\left(\partial_{0, \nu}^{-1}\right)$ a linear material-law. ${ }^{4}$ Moreover, as $M\left(\partial_{0, \nu}^{-1}\right)$ is a function of $\partial_{0, \nu}^{-1}$, we obtain the translation invariance of $M\left(\partial_{0, \nu}^{-1}\right)$, i.e. for each $h \in \mathbb{R}$ we have $\tau_{h} M\left(\partial_{0, \nu}^{-1}\right)=$ $M\left(\partial_{0, \nu}^{-1}\right) \tau_{h}$, where by $\tau_{h}$ we denote the translation operator on $H_{\nu, 0}(\mathbb{R} ; H)$ given by $\left(\tau_{h} u\right)(t):=u(t+h)$ for $u \in H_{\nu, 0}(\mathbb{R} ; H)$ and $t \in \mathbb{R}$.

\subsection{Maximal Monotone Relations}

We recall the notion of monotone and maximal monotone relations in Hilbert spaces. Moreover we state Minty's famous theorem, which gives a characterization of maximal monotonicity, which will be frequently used in the forthcoming parts. Additionally, we recall the definition of the Yosida-approximation of a maximal monotone relation and state a well-known perturbation result, which will be the key argument for proving our solution theory for inclusions of the form 1. Throughout let $H$ be a Hilbert space and $A \subseteq H \oplus H$ be a binary relation.

Definition. The relation $A$ is called monotone, if for each $(u, v),(x, y) \in A$ the inequality

$$
\operatorname{Re}\langle u-x \mid v-y\rangle \geq 0
$$

holds. We call $A$ maximal monotone, if $A$ is monotone and there exists no proper monotone extension of $A$, i.e. for each monotone relation $B \subseteq H \oplus H$ with $A \subseteq B$ it follows that $A=B$.

From this definition we can immediately derive the demi-closedness of maximal monotone relations, which in particular means that if $\left(u_{n}\right)_{n \in \mathbb{N}}$ and $\left(v_{n}\right)_{n \in \mathbb{N}}$

\footnotetext{
${ }^{4}$ The analyticity yields, employing a Paley-Wiener-type result (cf. [23]), the causality of the operator $M\left(\partial_{0, \nu}^{-1}\right)$.
} 
are sequences in $H$ such that $u_{n} \rightarrow u$ and $v_{n} \rightarrow v$ and $\left(u_{n}, v_{n}\right) \in A$ for each $n \in \mathbb{N}$, then $(u, v) \in A$.

Before we state Minty's Theorem, we introduce a linear structure on the set of binary relations. Let $H_{0}, H_{1}$ be two Hilbert spaces, $B, C \subseteq H_{0} \oplus H_{1}$ and $\lambda \in \mathbb{C}$. Then we define the relation $\lambda B+C \subseteq H_{0} \oplus H_{1}$ by

$\lambda B+C:=\left\{(u, v) \in H_{0} \oplus H_{1} \mid \exists x, y \in H_{1}:(u, x) \in B,(u, y) \in C, v=\lambda x+y\right\}$.

Moreover, for $M \subseteq H_{0}$ and $N \subseteq H_{1}$ we set

$$
\begin{aligned}
& B[M]:=\left\{y \in H_{1} \mid \exists x \in M:(x, y) \in B\right\}, \\
& {[N] B:=\left\{x \in H_{0} \mid \exists y \in N:(x, y) \in B\right\} .}
\end{aligned}
$$

For a monotone relation we can characterize the maximal monotonicity by using Minty's Theorem. A proof can be found for instance in [12],[1] or [26].

Theorem 2.1 (G. Minty, [12]). Let $A$ be monotone. Then the following statements are equivalent:

(i) $A$ is maximal monotone,

(ii) there exists $\lambda>0$ such that $(1+\lambda A)[H]=H$,

(iii) for each $\lambda>0$ it holds $(1+\lambda A)[H]=H$.

Remark 2.2. Let $A$ be maximal monotone.

(a) Due to the monotonicity of $A$, the relation $(1+\lambda A)^{-1}$ is right-unique for each $\lambda>0$, i.e. a mapping, whose domain equals $H$ according to 2.1. Indeed, one can show that $J_{\lambda}(A):=(1+\lambda A)^{-1}$ is Lipschitz-continuous with a Lipschitz-constant less than or equal to 1 (see e.g. [13, Theorem 1.3], [26, Proposition 1.12]). Moreover, we define $A_{\lambda}:=\lambda^{-1}\left(1-J_{\lambda}(A)\right)$ for $\lambda>0$ and obtain again a Lipschitz-continuous mapping with a Lipschitzconstant less than or equal to $\lambda^{-1}$. Furthermore, $A_{\lambda}$ is monotone for each $\lambda>0$. The mappings $\left(A_{\lambda}\right)_{\lambda>0}$ are called the Yosida-approximation of $A$. Moreover, for each $\lambda>0$ and $x \in H$ we have $\left(J_{\lambda}(A)(x), A_{\lambda}(x)\right) \in A$.

(b) For $x \in[H] A$ the set $A[\{x\}]$ is convex and closed. Hence, we can find the element in $A[\{x\}]$ possessing minimal norm, and we denote this element by $A^{0}(x)$. Then one can show, that for each $x \in[H] A$ and $\lambda>0$ the inequality $\left|A_{\lambda}(x)\right| \leq\left|A^{0}(x)\right|$ holds (see [13, Theorem 1.3], [26, Proposition 1.12]). 
Finally, we want to state two perturbation results for maximal monotone relations. If $A, B \subseteq H \oplus H$ are two monotone relations, then clearly $A+B$ is also monotone. However, in general the sum of two maximal monotone relations is not maximal monotone again. So the question is: If $A$ and $B$ are maximal monotone, when is $A+B$ maximal monotone? A positive answer can be given, if one of the relations is a Lipschitz-continuous mapping defined on the whole space $H$.

Lemma 2.3. Let $A$ be maximal monotone and $B: H \rightarrow H$ monotone and Lipschitz-continuous. Then $A+B$ is maximal monotone.

The proof is based on an application of the contraction mapping theorem and can be found for instance in [1, Lemme 2.4] or in [26, Lemma 1.15]. If $A$ and $B$ are maximal monotone relations in $H$, we can use the previous lemma and the fact that $B_{\lambda}$ is monotone and Lipschitz-continuous for each $\lambda>0$ to derive the following perturbation result.

Proposition 2.4. Let $A, B \subseteq H \oplus H$ be maximal monotone and $y \in H$. For $\lambda>0$ define $x_{\lambda}:=\left(1+A+B_{\lambda}\right)^{-1}(y)$. If $\sup _{\lambda>0}\left|B_{\lambda}\left(x_{\lambda}\right)\right|<\infty$, then $\left(x_{\lambda}\right)_{\lambda>0}$ converges to some $x \in H$ as $\lambda \rightarrow 0$ with $(x, y) \in 1+A+B$. In particular $A+B$ is maximal monotone.

For a proof of this statement we refer to [5, Lemma 7.41] or [26, Lemma $1.17]$.

\subsection{Abstract Boundary Data Spaces}

The last concept we need for our main results are so-called abstract boundary data spaces, introduced in [20]. Let $H_{0}, H_{1}$ be two Hilbert spaces and $G_{c} \subseteq$ $H_{0} \oplus H_{1}, D_{c} \subseteq H_{1} \oplus H_{0}$ be two densely defined closed linear operators. Moreover, assume that $G_{c}$ and $D_{c}$ are formally skew-adjoint, i.e. $G_{c} \subseteq\left(-D_{c}\right)^{*}=: G$, which also yields $D_{c} \subseteq\left(-G_{c}\right)^{*}=: D .{ }^{5}$ If we equip the domains of $G$ and $D$ with their respective graph-norms, we get that $\mathcal{D}\left(G_{c}\right)$ and $\mathcal{D}\left(D_{c}\right)$ are closed linear subspaces of $\mathcal{D}(G)$ and $\mathcal{D}(D)$ and hence, the projection theorem yields

$$
\begin{aligned}
& \mathcal{D}(G):=\mathcal{D}\left(G_{c}\right) \oplus \mathcal{D}\left(G_{c}\right)^{\perp}, \\
& \mathcal{D}(D):=\mathcal{D}\left(D_{c}\right) \oplus \mathcal{D}\left(D_{c}\right)^{\perp},
\end{aligned}
$$

\footnotetext{
${ }^{5}$ As a typical example one can think of $G_{c}$ and $D_{c}$ as the closure of the gradient and the divergence on $L_{2}$, defined on test-functions. Then the adjoints $G$ and $D$ are nothing but the usual weak gradient and weak divergence in $L_{2}$ (see Subsection 4.2).
} 
where the orthocomplements have to be taken with respect to the inner products induced by the graph norms of $G$ and $D$, respectively. An easy computation yields

$$
\begin{aligned}
& \mathcal{B D}(G):=\mathcal{D}\left(G_{c}\right)^{\perp}=[\{0\}](1-D G), \\
& \mathcal{B D}(D):=\mathcal{D}\left(D_{c}\right)^{\perp}=[\{0\}](1-G D) .
\end{aligned}
$$

The spaces $\mathcal{B D}(G)$ and $\mathcal{B D}(D)$ are called abstract boundary data spaces of $G$ and $D$, respectively. ${ }^{6}$ Clearly, $G[\mathcal{B D}(G)] \subseteq \mathcal{B D}(D)$ and $D[\mathcal{B D}(D)] \subseteq \mathcal{B D}(G)$. We define the operators

$$
\begin{aligned}
& \dot{G}: \mathcal{B D}(G) \rightarrow \mathcal{B D}(D), \\
& \dot{D}: \mathcal{B D}(D) \rightarrow \mathcal{B D}(G),
\end{aligned}
$$

as the respective restrictions of $G$ and $D$. As $\mathcal{B D}(G)$ and $\mathcal{B D}(D)$ are closed subspaces of $\mathcal{D}(G)$ and $\mathcal{D}(D)$, respectively, they inherit the Hilbert space structure from these supersets. With respect to these topologies, the operators $G$ and $D$ enjoy a surprising property.

Proposition 2.5 ([20, Theorem 5.2]). The operators $\dot{G}$ and $\dot{D}$ are unitary. Furthermore, $(\dot{G})^{*}=\dot{D}$ and so $(\dot{D})^{*}=\dot{G}$.

\section{Evolutionary Inclusions}

Let $H$ be a Hilbert space. In this section we study evolutionary inclusions of the form

$$
(u, f) \in \overline{\partial_{0, \nu} M\left(\partial_{0, \nu}^{-1}\right)+A},
$$

\footnotetext{
${ }^{6}$ In applications, where $G_{c}$ and $D_{c}$ will be defined as the closure of differential operators, defined on test functions, the elements of the domains of $G_{c}$ and $D_{c}$ can be interpreted as those elements in the domain of $G$ and $D$ with vanishing traces. For instance, if $G_{c}$ is the closure of the gradient defined on test-functions, then $\mathcal{D}\left(G_{c}\right)$ is the classical Sobolev-space $W_{2,0}^{1}$, i.e. the space of weakly differentiable functions in $L_{2}$ with vanishing traces. Hence, the boundary values of a function in $\mathcal{D}(G)$ just depend on the boundary values of its projection onto $\mathcal{B D}(G)$.
} 
where $M: B_{\mathbb{C}}\left(\frac{1}{2 \nu}, \frac{1}{2 \nu}\right) \rightarrow L(H)$ is a linear material law for some $\nu>0$ and $A \subseteq H_{\nu, 0}(\mathbb{R} ; H) \oplus H_{\nu, 0}(\mathbb{R} ; H)$ is an autonomous maximal monotone relation, i.e. $A$ is maximal monotone and for each $h \in \mathbb{R}$ and $(u, v) \in A$ it follows that $(u(\cdot+h), v(\cdot+h)) \in A$. The function $f \in H_{\nu, 0}(\mathbb{R} ; H)$ is a given source term and $u \in H_{\nu, 0}(\mathbb{R} ; H)$ is the unknown. We address the question of well-posedness of this problem, i.e. we show the uniqueness, existence and continuous dependence of a solution $u$ on the given data $f$. More precisely, we show that $\left(\overline{\partial_{0, \nu} M\left(\partial_{0, \nu}^{-1}\right)+A}\right)^{-1}$ is a Lipschitz-continuous mapping, whose domain is the whole space $H_{\nu, 0}(\mathbb{R} ; H)$. The second property we consider, is causality of the solution operator $\left(\overline{\partial_{0, \nu} M\left(\partial_{0, \nu}^{-1}\right)+A}\right)^{-1}$.

\subsection{Well-Posedness}

This subsection is devoted to the well-posedness of differential inclusions of the form 4. Throughout, let $H$ be a Hilbert space, $M: B_{\mathbb{C}}\left(\frac{1}{2 \nu}, \frac{1}{2 \nu}\right) \rightarrow L(H)$ a bounded strongly measurable function for some $\nu>0$ and $A \subseteq H_{\nu, 0}(\mathbb{R} ; H) \oplus$ $H_{\nu, 0}(\mathbb{R} ; H)$.

Hypotheses. We say that $M$ and $A$ satisfy the hypotheses (H1), (H2) and (H3) respectively, if

(H1) there exists $c>0$ such that for all $z \in B_{\mathbb{C}}\left(\frac{1}{2 \nu}, \frac{1}{2 \nu}\right)$ the inequality $\mathrm{R} z^{-1} M(z) \geq c$ holds.

(H2) $A$ is maximal monotone and autonomous, i.e. for every $h \in \mathbb{R}$ and $(u, v) \in$ $A$ we have $(u(\cdot+h), v(\cdot+h)) \in A$.

(H3) for all $(u, v),(x, y) \in A$ the estimate $\int_{-\infty}^{0} \operatorname{Re} u(t)-x(t)|v(t)-y(t)\rangle e^{-2 \nu t} \mathrm{~d} t$ $\geq 0$ holds.

\section{Remark 3.1.}

(a) A typical example for an autonomous maximal monotone relation is the extension of a maximal monotone relation $B \subseteq H \oplus H$, given by

$$
\begin{aligned}
A:=\left\{(u, v) \in H_{\nu, 0}(\mathbb{R} ; H) \oplus H_{\nu, 0}(\mathbb{R} ; H) \mid\right. & (u(t), v(t)) \in B \text { for almost every } t \in \mathbb{R}\} .
\end{aligned}
$$

If one assumes that $(0,0) \in B$, then $A$ is maximal monotone (cf. [13, p. $31])$. 
(b) In case of $A$ being the canonical extension of a skew-selfadjoint operator on $H$, the evolutionary problem 4 was originally considered by Picard in several works (cf. [15],[16] and [18]). The more general case of an autonomous operator $A: D(A) \subseteq H_{\nu, 0}(\mathbb{R} ; H) \rightarrow H_{\nu, 0}(\mathbb{R} ; H)$ was treated in [17].

(c) If $A$ is the extension of a maximal monotone relation $B \subseteq H \oplus H$ (compare (a)) and $M\left(\partial_{0, \nu}^{-1}\right)$ is of the particular form $M\left(\partial_{0, \nu}^{-1}\right)=M_{0}+\partial_{0, \nu}^{-1} M_{1}$, the problem was addressed by the author in [27]. Indeed, in [27] the inclusion was studied on the half line $\mathbb{R}_{\geq 0}$ instead of $\mathbb{R}$, which in particular implies that one can omit the additional assumption $(0,0) \in B$ in (a) in order to obtain the maximal monotonicity of the extension $A$.

First, we state the following simple, but useful observation.

Lemma 3.2. Let $\nu>0$ and $T \in L\left(H_{\nu, 0}(\mathbb{R} ; H)\right)$. Assume that $T \partial_{0, \nu} \subseteq$ $\partial_{0, \nu} T$. Then $\mathcal{D}\left(\partial_{0, \nu}\right)$ is a core of $\partial_{0, \nu} T$ and $\left(\partial_{0, \nu} T\right)^{*}=\partial_{0, \nu}^{*} T^{*}$.

Proof. Let $u \in \mathcal{D}\left(\partial_{0, \nu} T\right)$, i.e. $T u \in \mathcal{D}\left(\partial_{0, \nu}\right)$. For $\varepsilon>0$ we define $u_{\varepsilon}:=$ $\left(1+\varepsilon \partial_{0, \nu}\right)^{-1} u \in \mathcal{D}\left(\partial_{0, \nu}\right)$ and we obtain that $u_{\varepsilon} \rightarrow u$ as $\varepsilon \rightarrow 0$ as well as

$$
\partial_{0, \nu} T u_{\varepsilon}=\partial_{0, \nu} T\left(1+\varepsilon \partial_{0, \nu}\right)^{-1} u=\left(1+\varepsilon \partial_{0, \nu}\right)^{-1} \partial_{0, \nu} T u \rightarrow \partial_{0, \nu} T u \quad(\varepsilon \rightarrow 0) .
$$

In particular, this yields that $\partial_{0, \nu} T=\overline{T \partial_{0, \nu}}$ and hence,

$$
\left(\partial_{0, \nu} T\right)^{*}=\left(T \partial_{0, \nu}\right)^{*}=\partial_{0, \nu}^{*} T^{*} .
$$

Lemma 3.3. Assume that $M$ satisfies (H1). Then $\partial_{0, \nu} M\left(\partial_{0, \nu}^{-1}\right)-c$ is a maximal monotone operator in $H_{\nu, 0}(\mathbb{R} ; H)$.

Proof. Using the unitary equivalence of $\partial_{0, \nu} M\left(\partial_{0, \nu}^{-1}\right)$ in $H_{\nu, 0}(\mathbb{R} ; H)$ and

$$
(\mathrm{i} m+\nu) M\left(\frac{1}{\mathrm{i} m+\nu}\right)
$$

in $L_{2}(\mathbb{R} ; H)$, where $m$ denotes the multiplication-by-the-argument operator (see Subsection 2.1), it suffices to prove the maximal monotonicity of

$$
(\mathrm{i} m+\nu) M\left(\frac{1}{\mathrm{i} m+\nu}\right)-c
$$

in $L_{2}(\mathbb{R} ; H)$. The monotonicity follows immediately from (H1). Furthermore, by (H1) we get the strict monotonicity of

$$
\left((\mathrm{i} m+\nu) M\left(\frac{1}{\mathrm{i} m+\nu}\right)\right)^{*}=(-\mathrm{i} m+\nu) M\left(\frac{1}{\mathrm{i} m+\nu}\right)^{*},
$$


where for $u \in L_{2}(\mathbb{R} ; H)$ and almost every $t \in \mathbb{R}$ we have that

$$
\left(M\left(\frac{1}{\mathrm{i} m+\nu}\right)^{*} u\right)(t)=M\left(\frac{1}{\mathrm{i} t+\nu}\right)^{*} u(t) .
$$

The latter implies that the operator $(\mathrm{i} m+\nu) M\left(\frac{1}{\mathrm{i} m+\nu}\right)$ has a dense range, which gives, using the closedness and the continuous invertibility of the operator, that $(\mathrm{i} m+\nu) M\left(\frac{1}{\mathrm{i} m+\nu}\right)$ is onto. Hence 2.1 yields the assertion.

Proposition 3.4. Let $B \subseteq H_{\nu, 0}(\mathbb{R} ; H) \oplus H_{\nu, 0}(\mathbb{R} ; H)$ be monotone and let $M$ satisfy (H1). Then for each $(u, f),(v, g) \in \partial_{0, \nu} M\left(\partial_{0, \nu}^{-1}\right)+B$ the estimate

$$
|u-v|_{H_{\nu, 0}(\mathbb{R} ; H)} \leq \frac{1}{c}|f-g|_{H_{\nu, 0}(\mathbb{R} ; H)}
$$

holds.

Proof. Since the operator $\partial_{0, \nu} M\left(\partial_{0, \nu}^{-1}\right)-c$ is monotone, according to 3.3, we obtain that $\partial_{0, \nu} M\left(\partial_{0, \nu}^{-1}\right)-c+B$ is monotone. An easy argument, using the Cauchy-Schwarz-inequality, yields the assertion.

Remark 3.5. 3.4 especially yields the uniqueness and continuous dependence of a solution $u$ of 4 on the given right hand side $f$.

The next proposition yields the existence of a solution of 4 for every right hand side $f \in H_{\nu, 0}(\mathbb{R} ; H)$. The proof uses the perturbation result given in 2.4 in order to show the existence of a solution for $f \in C_{c}^{\infty}(\mathbb{R} ; H)$ and follows the same strategy as the one in [27, Proposition 4.6].

Proposition 3.6. Let $M$ satisfy (H1) and $A$ satisfy (H2). Then for each $f \in C_{c}^{\infty}(\mathbb{R} ; H)$ there exists $u \in H_{\nu, 0}(\mathbb{R} ; H)$ such that

$$
(u, f) \in \partial_{0, \nu} M\left(\partial_{0, \nu}^{-1}\right)+A .
$$

Proof. We replace $A$ by its Yosida approximation $A_{\lambda}$ for $\lambda>0$, which is a Lipschitz-continuous monotone mapping on $H_{\nu, 0}(\mathbb{R} ; H)$. Then, using 3.3 and 2.3 we find an element $u_{\lambda} \in H_{\nu, 0}(\mathbb{R} ; H)$ such that

$$
\left(u_{\lambda}, \frac{1}{c} f\right) \in 1+\frac{1}{c}\left(\partial_{0, \nu} M\left(\partial_{0, \nu}^{-1}\right)-c+A_{\lambda}\right),
$$


which is equivalent to

$$
\left(u_{\lambda}, f\right) \in \partial_{0, \nu} M\left(\partial_{0, \nu}^{-1}\right)+A_{\lambda}
$$

We prove that $\left(A_{\lambda}\left(u_{\lambda}\right)\right)_{\lambda>0}$ is uniformly bounded. For that purpose let $h>$ 0 . Then, using that $A_{\lambda}$ and $\partial_{0, \nu} M\left(\partial_{0, \nu}^{-1}\right)$ commute with the operator $\tau_{h}$ : $H_{\nu, 0}(\mathbb{R} ; H) \rightarrow H_{\nu, 0}(\mathbb{R} ; H)$ given by $\left(\tau_{h} v\right)(t):=v(t+h)$ for $t \in \mathbb{R}, v \in H_{\nu, 0}(\mathbb{R} ; H)$, we obtain that

$$
\left(\tau_{h} u_{\lambda}, \tau_{h} f\right) \in \partial_{0, \nu} M\left(\partial_{0, \nu}^{-1}\right)+A_{\lambda} .
$$

Since $A_{\lambda}$ is monotone, 3.4 yields

$$
\left|\tau_{h} u_{\lambda}-u_{\lambda}\right|_{H_{\nu, 0}(\mathbb{R} ; H)} \leq \frac{1}{c}\left|\tau_{h} f-f\right|_{H_{\nu, 0}(\mathbb{R} ; H)}
$$

and hence, using the mean-value inequality

$$
\left|\frac{1}{h}\left(\tau_{h} u_{\lambda}-u_{\lambda}\right)\right|_{H_{\nu, 0}(\mathbb{R} ; H)} \leq \frac{1}{c}\left|f^{\prime}\right|_{\infty} \sqrt{\mu_{\nu}(\operatorname{supp} f)}
$$

for each $h>0$, where supp $f$ denotes the support of $f$. The latter gives, by choosing a weak-convergent subsequence of $\left(\frac{1}{h}\left(\tau_{h} u_{\lambda}-u_{\lambda}\right)\right)_{h>0}$, that $u_{\lambda} \in$ $D\left(\partial_{0, \nu}\right)$ and that

$$
\left|\partial_{0, \nu} u_{\lambda}\right|_{H_{\nu, 0}(\mathbb{R} ; H)} \leq \frac{1}{c}\left|f^{\prime}\right|_{\infty} \sqrt{\mu_{\nu}(\operatorname{supp} f)}
$$

Hence, using 5 we obtain

$$
\begin{aligned}
\left|A_{\lambda}\left(u_{\lambda}\right)\right|_{H_{\nu, 0}(\mathbb{R} ; H)} & =\left|f-\partial_{0, \nu} M\left(\partial_{0, \nu}^{-1}\right) u_{\lambda}\right|_{H_{\nu, 0}(\mathbb{R} ; H)} \\
& \leq|f|_{H_{\nu, 0}(\mathbb{R} ; H)}+\left\|M\left(\partial_{0, \nu}^{-1}\right)\right\| \frac{1}{c}\left|f^{\prime}\right|_{\infty} \sqrt{\mu_{\nu}(\operatorname{supp} f)}
\end{aligned}
$$

for each $\lambda>0$. Thus, 2.4 applies and hence, we find an element $u \in H_{\nu, 0}(\mathbb{R} ; H)$ satisfying

$$
\left(u, \frac{1}{c} f\right) \in 1+\frac{1}{c}\left(\partial_{0, \nu} M\left(\partial_{0, \nu}^{-1}\right)-c+A\right),
$$

which gives

$$
(u, f) \in \partial_{0, \nu} M\left(\partial_{0, \nu}^{-1}\right)+A
$$

We summarize our findings of this subsection in the following theorem. 
Theorem 3.7 (Well-posedness of evolutionary inclusions). Let $H$ be a Hilbert space, $M: B_{\mathbb{C}}\left(\frac{1}{2 \nu}, \frac{1}{2 \nu}\right) \rightarrow L(H)$ a bounded strongly measurable function for some $\nu>0$ satisfying (H1) and $A \subseteq H_{\nu, 0}(\mathbb{R} ; H) \oplus H_{\nu, 0}(\mathbb{R} ; H)$ a relation satisfying (H2). Then for each $f \in H_{\nu, 0}(\mathbb{R} ; H)$ there exists a unique $u \in H_{\nu, 0}(\mathbb{R} ; H)$ such that

$$
(u, f) \in \overline{\partial_{0, \nu} M\left(\partial_{0, \nu}^{-1}\right)+A} .
$$

Moreover, $\left(\overline{\partial_{0, \nu} M\left(\partial_{0, \nu}^{-1}\right)+A}\right)^{-1}$ is Lipschitz-continuous with a Lipschitz constant less than or equal to $\frac{1}{c}$.

\subsection{Causality}

In this subsection we prove the causality of the solution operator

$$
\left(\overline{\partial_{0, \nu} M\left(\partial_{0, \nu}^{-1}\right)+A}\right)^{-1}
$$

of an inclusion of the form 4. First we give an equivalent condition for the causality of the operator $\left(\partial_{0, \nu} M\left(\partial_{0, \nu}^{-1}\right)\right)^{-1}$, which will enable us to prove the causality for the case of non-vanishing $A$. The statement was already given in [26, Proposition 2.65] in a slightly more general version. However, for sake of completeness we present the proof.

Lemma 3.8. Let $\nu>0$ and $M: B_{\mathbb{C}}\left(\frac{1}{2 \nu}, \frac{1}{2 \nu}\right) \rightarrow L(H)$ be a strongly measurable bounded mapping satisfying the hypothesis (H1). Then the following statements are equivalent:

(i) the operator $\left(\partial_{0, \nu} M\left(\partial_{0, \nu}^{-1}\right)\right)^{-1}: H_{\nu, 0}(\mathbb{R} ; H) \rightarrow H_{\nu, 0}(\mathbb{R} ; H)$ is causal,

(ii) for every $u \in \mathcal{D}\left(\partial_{0, \nu} M\left(\partial_{0, \nu}^{-1}\right)\right)$ we have that

$$
\int_{-\infty}^{0} \operatorname{Re}\left\langle\partial_{0, \nu} M\left(\partial_{0, \nu}^{-1}\right) u(t) \mid u(t)\right\rangle e^{-2 \nu t} \mathrm{~d} t \geq c \int_{-\infty}^{0}\langle u(t) \mid u(t)\rangle e^{-2 \nu t} \mathrm{~d} t .
$$

Proof. Assume that $\left(\partial_{0, \nu} M\left(\partial_{0, \nu}^{-1}\right)\right)^{-1}$ is causal and let

$$
u \in \mathcal{D}\left(\partial_{0, \nu} M\left(\partial_{0, \nu}^{-1}\right)\right) .
$$


We set $v:=\partial_{0, \nu} M\left(\partial_{0, \nu}^{-1}\right) u$ and obtain

$$
\begin{aligned}
& \int_{-\infty}^{0} \operatorname{Re}\left\langle\partial_{0, \nu} M\left(\partial_{0, \nu}^{-1}\right) u(t) \mid u(t)\right\rangle e^{-2 \nu t} \mathrm{~d} t \\
= & \int_{-\infty}^{0} \operatorname{Re}\left\langle v(t) \mid\left(\left(\partial_{0, \nu} M\left(\partial_{0, \nu}^{-1}\right)\right)^{-1} v\right)(t)\right\rangle e^{-2 \nu t} \mathrm{~d} t \\
= & \left.\operatorname{Re}\left\langle v \mid \chi_{(-\infty, 0]}(m)\left(\partial_{0, \nu} M\left(\partial_{0, \nu}^{-1}\right)\right)^{-1} v\right\rangle_{H_{\nu, 0}(\mathbb{R} ; H)}\left(\chi_{(-\infty, 0]}(m) v\right)\right\rangle_{H_{\nu, 0}(\mathbb{R} ; H)} \\
= & \operatorname{Re}\left\langle v \mid \chi_{(-\infty, 0]}(m)\left(\partial_{0, \nu} M\left(\partial_{0, \nu}^{-1}\right)\right)^{-1}\left(\partial_{0, \nu} M\left(\partial_{0, \nu}^{-1}\right)\right)^{-1}\left(\chi_{(-\infty, 0]}(m) v\right)\right\rangle_{H_{\nu, 0}(\mathbb{R} ; H)} \\
= & \operatorname{Re}\left\langle\chi_{(-\infty, 0]}(m) v\right|\left(\partial_{0}\right.
\end{aligned}
$$

Using 3.3 we get that

$$
\begin{aligned}
& \int_{-\infty}^{0} \operatorname{Re}\left\langle\partial_{0, \nu} M\left(\partial_{0, \nu}^{-1}\right) u(t) \mid u(t)\right\rangle e^{-2 \nu t} \mathrm{~d} t \\
& =\operatorname{Re}\left\langle\chi_{(-\infty, 0]}(m) v \mid\left(\partial_{0, \nu} M\left(\partial_{0, \nu}^{-1}\right)\right)^{-1}\left(\chi_{(-\infty, 0]}(m) v\right)\right\rangle_{H_{\nu, 0}(\mathbb{R} ; H)} \\
& \geq c\left|\left(\partial_{0, \nu} M\left(\partial_{0, \nu}^{-1}\right)\right)^{-1}\left(\chi_{(-\infty, 0]}(m) v\right)\right|_{H_{\nu, 0}(\mathbb{R} ; H)}^{2} \\
& \geq c\left|\chi_{(-\infty, 0]}(m)\left(\partial_{0, \nu} M\left(\partial_{0, \nu}^{-1}\right)\right)^{-1}\left(\chi_{(-\infty, 0]}(m) v\right)\right|_{H_{\nu, 0}(\mathbb{R} ; H)}^{2} \\
& =c\left|\chi_{(-\infty, 0]}(m)\left(\partial_{0, \nu} M\left(\partial_{0, \nu}^{-1}\right)\right)^{-1} v\right|_{H_{\nu, 0}(\mathbb{R} ; H)}^{2} \\
& =c \int_{-\infty}^{0}|u(t)|^{2} e^{-2 \nu t} \mathrm{~d} t .
\end{aligned}
$$

Assume now that (ii) holds. Using that $\partial_{0, \nu} M\left(\partial_{0, \nu}^{-1}\right)$ is translation invariant, i.e. it commutes with the translation operator $\tau_{h}$ for each $h \in \mathbb{R}$, the asserted 
inequality implies

$$
\int_{-\infty}^{a} \operatorname{Re}\left\langle\partial_{0, \nu} M\left(\partial_{0, \nu}^{-1}\right) u(t) \mid u(t)\right\rangle e^{-2 \nu t} \mathrm{~d} t \geq c \int_{-\infty}^{a}|u(t)|^{2} e^{-2 \nu t} \mathrm{~d} t
$$

for each $a \in \mathbb{R}, u \in \mathcal{D}\left(\partial_{0, \nu} M\left(\partial_{0, \nu}^{-1}\right)\right)$. Due to the linearity of $\left(\partial_{0, \nu} M\left(\partial_{0, \nu}^{-1}\right)\right)^{-1}$ it suffices to prove that $\chi_{(-\infty, a]}(m) f=0$ implies

$$
\chi_{(-\infty, a]}(m)\left(\partial_{0, \nu} M\left(\partial_{0, \nu}^{-1}\right)\right)^{-1} f=0
$$

for each $f \in H_{\nu, 0}(\mathbb{R} ; H)$. So let $f \in H_{\nu, 0}(\mathbb{R} ; H)$ with $\chi_{(-\infty, a]}(m) f=0$ for some $a \in \mathbb{R}$ and define $u:=\left(\partial_{0, \nu} M\left(\partial_{0, \nu}^{-1}\right)\right)^{-1} f$. Then 6 gives

$$
c \int_{-\infty}^{a}|u(t)|^{2} e^{-2 \nu t} \mathrm{~d} t \leq \int_{-\infty}^{a} \operatorname{Re}\langle f(t) \mid u(t)\rangle e^{-2 \nu t} \mathrm{~d} t=0,
$$

which implies $\chi_{(-\infty, a]}(m) u=0$.

Remark 3.9. If $M: B_{\mathbb{C}}\left(\frac{1}{2 \nu}, \frac{1}{2 \nu}\right) \rightarrow L(H)$ is a linear material law and satisfies the hypothesis (H1), then 3.8 gives that 6 holds for every $u \in$ $\mathcal{D}\left(\partial_{0, \nu} M\left(\partial_{0, \nu}^{-1}\right)\right)$ and every $a \in \mathbb{R}$, since the assumed analyticity for $M$ yields the causality of $\left(\partial_{0, \nu} M\left(\partial_{0, \nu}^{-1}\right)\right)^{-1}$ (see [15, Lemma 2.5]).

Now we are able to show the causality of the solution operator associated to the evolutionary inclusion 4 .

Proposition 3.10 (Causality of evolutionary inclusions). Let $H$ be a Hilbert space, $\nu>0$ and $M: B_{\mathbb{C}}\left(\frac{1}{2 \nu}, \frac{1}{2 \nu}\right) \rightarrow L(H)$ a linear material law, satisfying hypothesis (H1). Furthermore let $A \subseteq H_{\nu, 0}(\mathbb{R} ; H) \oplus H_{\nu, 0}(\mathbb{R} ; H)$ be a binary relation satisfying $(\mathrm{H} 2)$ and $(\mathrm{H} 3)$. Then, the solution operator

$$
\left(\overline{\partial_{0, \nu} M\left(\partial_{0, \nu}^{-1}\right)+A}\right)^{-1}: H_{\nu, 0}(\mathbb{R} ; H) \rightarrow H_{\nu, 0}(\mathbb{R} ; H),
$$

which exists according to 3.7, is causal.

Proof. First note that due to the translation invariance of $A$ and $\partial_{0, \nu} M\left(\partial_{0, \nu}^{-1}\right)$ it suffices to check that for $f, g \in H_{\nu, 0}(\mathbb{R} ; H)$ satisfying $\chi_{(-\infty, 0]}(m)(f-g)=0$ 
we have that

$$
\chi_{(-\infty, 0]}(m)\left(\left(\overline{\partial_{0, \nu} M\left(\partial_{0, \nu}^{-1}\right)+A}\right)^{-1}(f)-\left(\overline{\partial_{0, \nu} M\left(\partial_{0, \nu}^{-1}\right)+A}\right)^{-1}(g)\right)=0 .
$$

So let $f, g \in H_{\nu, 0}(\mathbb{R} ; H)$ with $\chi_{(-\infty, 0]}(m)(f-g)=0$. We choose sequences $\left(f_{n}\right)_{n \in \mathbb{N}}$ and $\left(g_{n}\right)_{n \in \mathbb{N}}$ in $C_{c}^{\infty}(\mathbb{R} ; H) \subseteq\left(\partial_{0, \nu} M\left(\partial_{0, \nu}^{-1}\right)+A\right)\left[H_{\nu, 0}(\mathbb{R} ; H)\right]($ see 3.6) such that $f_{n} \rightarrow f$ and $g_{n} \rightarrow g$ in $H_{\nu, 0}(\mathbb{R} ; H)$ as $n \rightarrow \infty$. For $n \in \mathbb{N}$ we define

$$
\begin{aligned}
& u_{n}:=\left(\partial_{0, \nu} M\left(\partial_{0, \nu}^{-1}\right)+A\right)^{-1}\left(f_{n}\right), \\
& v_{n}:=\left(\partial_{0, \nu} M\left(\partial_{0, \nu}^{-1}\right)+A\right)^{-1}\left(g_{n}\right),
\end{aligned}
$$

and due to the continuity of

$$
\left(\overline{\partial_{0, \nu} M\left(\partial_{0, \nu}^{-1}\right)+A}\right)^{-1}
$$

we get that

$$
u_{n} \rightarrow\left(\overline{\partial_{0, \nu} M\left(\partial_{0, \nu}^{-1}\right)+A}\right)^{-1}(f)
$$

as well as

$$
v_{n} \rightarrow\left(\overline{\partial_{0, \nu} M\left(\partial_{0, \nu}^{-1}\right)+A}\right)^{-1}(g)
$$

in $H_{\nu, 0}(\mathbb{R} ; H)$. We estimate, using 3.8 and (H3)

$$
\int_{-\infty}^{0} \operatorname{Re}\left(u_{n}(t)-v_{n}(t)\left|f_{n}(t)-g_{n}(t)\right\rangle e^{-2 \nu t} \mathrm{~d} t \geq c \int_{-\infty}^{0}\left|u_{n}(t)-v_{n}(t)\right|^{2} e^{-2 \nu t} \mathrm{~d} t\right.
$$

for each $n \in \mathbb{N}$. Passing to the limit as $n \rightarrow \infty$ gives

$$
\int_{-\infty}^{0}\left|\left(\overline{\partial_{0, \nu} M\left(\partial_{0, \nu}^{-1}\right)+A}\right)^{-1}(f)-\left(\overline{\partial_{0, \nu} M\left(\partial_{0, \nu}^{-1}\right)+A}\right)^{-1}(g)\right|^{2} e^{-2 \nu t} \mathrm{~d} t \leq 0
$$

which yields the causality. 


\section{Nonlinear Boundary Conditions}

In this subsection we study a class of evolutionary equations, which covers evolutionary problems with nonlinear boundary conditions, modelled by an inclusion on the boundary. Those equations occur for instance in the study of problems in elasticity with frictional boundary conditions, where the behaviour on the boundary is described by variational inequalities (cf. [4],[25] and [14]) or by suitable differential inclusions (cf. [9],[11] and [10]). Moreover, since we consider operators acting in time and space, also problems with boundary conditions given by ordinary differential equations, delay equations or inclusions can be treated within our framework.

Throughout let $H_{0}$ and $H_{1}$ be Hilbert spaces and $G_{c} \subseteq H_{0} \oplus H_{1}$ and $D_{c} \subseteq$ $H_{1} \oplus H_{0}$ two densely defined closed linear operators. Moreover assume that $G_{c}$ and $D_{c}$ are formally skew-adjoint, i.e.

$$
\begin{aligned}
& G_{c} \subseteq-\left(D_{c}\right)^{*}=: G, \\
& D_{c} \subseteq-\left(G_{c}\right)^{*}=: D .
\end{aligned}
$$

We do not distinguish notationally between the operators $G_{c}, D_{c}, G$ and $D$ and their canonical extensions to $H_{\nu, 0}\left(\mathbb{R} ; H_{0}\right)$ and $H_{\nu, 0}\left(\mathbb{R} ; H_{1}\right)$, respectively. Recall from Subsection 2.3 that

$$
\begin{aligned}
& \mathcal{B D}(G):=\mathcal{D}\left(G_{c}\right)^{\perp}=[\{0\}](1-D G), \\
& \mathcal{B D}(D):=\mathcal{D}\left(D_{c}\right)^{\perp}=[\{0\}](1-G D),
\end{aligned}
$$

where the orthocomplements are taken with respect to the inner products in $\mathcal{D}(G)$ and $\mathcal{D}(D)$, respectively. We consider the following operator $A: \mathcal{D}(A) \subseteq$ $H_{\nu, 0}\left(\mathbb{R} ; H_{0} \oplus H_{1}\right) \rightarrow H_{\nu, 0}\left(\mathbb{R} ; H_{0} \oplus H_{1}\right)$ given by

$$
\begin{aligned}
\mathcal{D}(A) & :=\left\{(u, v) \in H_{\nu, 0}(\mathbb{R} ; \mathcal{D}(G) \oplus \mathcal{D}(D)) \mid\left(\pi_{\mathcal{B D}(G)} u, \dot{D} \pi_{\mathcal{B D}(D)} v\right) \in h\right\}, \\
A\left(\begin{array}{l}
u \\
v
\end{array}\right) & :=\left(\begin{array}{cc}
0 & D \\
G & 0
\end{array}\right)\left(\begin{array}{l}
u \\
v
\end{array}\right) .
\end{aligned}
$$

Here $h \subseteq H_{\nu, 0}(\mathbb{R} ; \mathcal{B D}(G)) \oplus H_{\nu, 0}(\mathbb{R} ; \mathcal{B D}(G))$ is a binary relation. We will show that under appropriate assumptions on $h$, the operator $A$ satisfies the hypotheses (H2) and (H3) and thus 3.7 and 3.10 are applicable. The first subsection is devoted to the proof of the following theorem. 
Theorem 4.1. If $h$ satisfies the hypotheses (H2) or (H3), then so does $A$.

In Subsection 4.2 we will illustrate the applicability of the abstract class of evolutionary inclusions of the form 4 , where the relation $A$ is an operator of the form given in 7 .

\subsection{The Proof of Theorem 4.1.}

We begin to show the monotonicity of $A$ under the assumption that $h$ is monotone. Indeed, we will show an equality, which will imply, despite the monotonicity of $A$, that $A$ satisfies (H3) if $h$ does.

Lemma 4.2. Let $(u, v),(x, y) \in \mathcal{D}(A)$. Then for each $a \in \mathbb{R}$ the following equality holds:

$$
\begin{aligned}
& \operatorname{Re} \int_{-\infty}^{a}\left\langle A\left(\begin{array}{l}
u \\
v
\end{array}\right)(t)-A\left(\begin{array}{l}
x \\
y
\end{array}\right)(t) \mid\left(\begin{array}{l}
u \\
v
\end{array}\right)(t)-\left(\begin{array}{l}
x \\
y
\end{array}\right)(t)\right\rangle_{H_{0} \oplus H_{1}} e^{-2 \nu t} \mathrm{~d} t \\
& =\operatorname{Re} \int_{-\infty}^{a}\left\langle\pi_{\mathcal{B D}(G)}(u(t)-x(t)) \mid \stackrel{\bullet}{D} \pi_{\mathcal{B D}(D)}(v(t)-y(t))\right\rangle_{\mathcal{B D}(G)} e^{-2 \nu t} \mathrm{~d} t .
\end{aligned}
$$

In particular, if $h$ is monotone, then so is $A$.

Proof. Let $a \in \mathbb{R}$. Then we compute

$$
\begin{aligned}
& \operatorname{Re} \int_{-\infty}^{a}\left\langle A\left(\begin{array}{l}
u \\
v
\end{array}\right)(t)-A\left(\begin{array}{l}
x \\
y
\end{array}\right)(t) \mid\left(\begin{array}{l}
u \\
v
\end{array}\right)(t)-\left(\begin{array}{l}
x \\
y
\end{array}\right)(t)\right\rangle_{H_{0} \oplus H_{1}} e^{-2 \nu t} \mathrm{~d} t \\
& =\operatorname{Re} \int_{-\infty}^{a}\langle D(v(t)-y(t)) \mid u(t)-x(t)\rangle_{H_{0}} e^{-2 \nu t} \mathrm{~d} t \\
& +\operatorname{Re} \int_{-\infty}^{a}\langle G(u(t)-x(t)) \mid v(t)-y(t)\rangle_{H_{1}} e^{-2 \nu t} \mathrm{~d} t \\
& =\operatorname{Re} \int_{-\infty}^{a}\langle D(v(t)-y(t)) \mid u(t)-x(t)\rangle_{H_{0}} e^{-2 \nu t} \mathrm{~d} t \\
& +\operatorname{Re} \int_{-\infty}^{a}\left\langle G P_{\mathcal{B D}(G)}(u(t)-x(t)) \mid v(t)-y(t)\right\rangle_{H_{1}} e^{-2 \nu t} \mathrm{~d} t
\end{aligned}
$$




$$
\begin{aligned}
& +\operatorname{Re} \int_{-\infty}^{a}\left\langle G_{c}\left(1-P_{\mathcal{B D}(G)}\right)(u(t)-x(t)) \mid v(t)-y(t)\right\rangle_{H_{1}} e^{-2 \nu t} \mathrm{~d} t \\
& =\operatorname{Re} \int_{-\infty}^{a}\langle D(v(t)-y(t)) \mid u(t)-x(t)\rangle_{H_{0}} e^{-2 \nu t} \mathrm{~d} t \\
& +\mathrm{Re} \int_{-\infty}^{a}\left\langle G P_{\mathcal{B D}(G)}(u(t)-x(t)) \mid v(t)-y(t)\right\rangle_{H_{1}} e^{-2 \nu t} \mathrm{~d} t \\
& -\operatorname{Re} \int_{-\infty}^{a}\left\langle\left(1-P_{\mathcal{B D}(G)}\right)(u(t)-x(t)) \mid D(v(t)-y(t))\right\rangle_{H_{0}} e^{-2 \nu t} \mathrm{~d} t \\
& =\operatorname{Re} \int_{-\infty}^{a}\left\langle G P_{\mathcal{B D}(G)}(u(t)-x(t)) \mid v(t)-y(t)\right\rangle_{H_{1}} e^{-2 \nu t} \mathrm{~d} t \\
& +\mathrm{Re} \int_{-\infty}^{a}\left\langle P_{\mathcal{B D}(G)}(u(t)-x(t)) \mid D(v(t)-y(t))\right\rangle_{H_{0}} e^{-2 \nu t} \mathrm{~d} t \\
& =\operatorname{Re} \int_{-\infty}^{a}\left\langle G P_{\mathcal{B D}(G)}(u(t)-x(t)) \mid P_{\mathcal{B D}(D)}(v(t)-y(t))\right\rangle_{H_{1}} e^{-2 \nu t} \mathrm{~d} t \\
& +\operatorname{Re} \int_{-\infty}^{a}\left\langle G P_{\mathcal{B D}(G)}(u(t)-x(t)) \mid\left(1-P_{\mathcal{B D}(D)}\right)(v(t)-y(t))\right\rangle_{H_{1}} e^{-2 \nu t} \mathrm{~d} t \\
& +\operatorname{Re} \int_{-\infty}^{a}\left\langle P_{\mathcal{B D}(G)}(u(t)-x(t)) \mid D P_{\mathcal{B D}(D)}(v(t)-y(t))\right\rangle_{H_{0}} e^{-2 \nu t} \mathrm{~d} t \\
& +\mathrm{Re} \int_{-\infty}^{a}\left\langle P_{\mathcal{B D}(G)}(u(t)-x(t)) \mid D_{c}\left(1-P_{\mathcal{B D}(D)}\right)(v(t)-y(t))\right\rangle_{H_{0}} e^{-2 \nu t} \mathrm{~d} t \\
& =\operatorname{Re} \int_{-\infty}^{a}\left\langle G P_{\mathcal{B D}(G)}(u(t)-x(t)) \mid P_{\mathcal{B D}(D)}(v(t)-y(t))\right\rangle_{H_{1}} e^{-2 \nu t} \mathrm{~d} t \\
& +\mathrm{Re} \int_{-\infty}^{a}\left\langle P_{\mathcal{B D}(G)}(u(t)-x(t)) \mid D P_{\mathcal{B D}(D)}(v(t)-y(t))\right\rangle_{H_{0}} e^{-2 \nu t} \mathrm{~d} t
\end{aligned}
$$




$$
\begin{aligned}
= & \operatorname{Re} \int_{-\infty}^{a}\left\langle G P_{\mathcal{B D}(G)}(u(t)-x(t)) \mid G D P_{\mathcal{B D}(D)}(v(t)-y(t))\right\rangle_{H_{1}} e^{-2 \nu t} \mathrm{~d} t \\
& +\operatorname{Re} \int_{-\infty}^{a}\left\langle P_{\mathcal{B D}(G)}(u(t)-x(t)) \mid D P_{\mathcal{B D}(D)}(v(t)-y(t))\right\rangle_{H_{0}} e^{-2 \nu t} \mathrm{~d} t \\
= & \operatorname{Re} \int_{-\infty}^{a}\left\langle P_{\mathcal{B D}(G)}(u(t)-x(t)) \mid D P_{\mathcal{B D}(D)}(v(t)-y(t))\right\rangle_{\mathcal{D}(G)} e^{-2 \nu t} \mathrm{~d} t \\
= & \operatorname{Re} \int_{-\infty}^{a}\left\langle\pi_{\mathcal{B D}(G)}(u(t)-x(t)) \mid \dot{D}_{\mathcal{B D}(D)}(v(t)-y(t))\right\rangle_{\mathcal{B D}(G)} e^{-2 \nu t} \mathrm{~d} t .
\end{aligned}
$$

Lemma 4.3. If the relation $h$ is closed, then so is $A$.

Proof. Let $\left(\left(u_{n}, v_{n}\right)\right)_{n \in \mathbb{N}}$ be a sequence in $\mathcal{D}(A)$ such that $u_{n} \rightarrow u, v_{n} \rightarrow v$ as well as $D v_{n} \rightarrow w$ and $G u_{n} \rightarrow x$ in $H_{\nu, 0}\left(\mathbb{R} ; H_{0}\right)$ and $H_{\nu, 0}\left(\mathbb{R} ; H_{1}\right)$ respectively. Due to the closedness of $G$ and $D$ we obtain that $u \in H_{\nu, 0}(\mathbb{R} ; \mathcal{D}(G)), v \in$ $H_{\nu, 0}(\mathbb{R} ; \mathcal{D}(D))$ and $G u=x, D v=w$. Note that this means that $u_{n} \rightarrow u$ and $v_{n} \rightarrow v$ in $H_{\nu, 0}(\mathbb{R} ; \mathcal{D}(G))$ and $H_{\nu, 0}(\mathbb{R} ; \mathcal{D}(D))$, respectively. The latter implies $\dot{D} \pi_{\mathcal{B D}(D)} v_{n} \rightarrow \stackrel{\bullet}{D} \pi_{\mathcal{B D}(D)} v$ and $\pi_{\mathcal{B D}(G)} u_{n} \rightarrow \pi_{\mathcal{B D}(G)} u$ in $H_{\nu, 0}(\mathbb{R} ; \mathcal{B D}(G))$. Since $\left(\pi_{\mathcal{B D}(G)} u_{n}, \stackrel{\bullet}{D} \pi_{\mathcal{B D}(D)} v_{n}\right) \in h$ for each $n \in \mathbb{N}$, the closedness of $h$ yields $\left(\pi_{\mathcal{B D}(G)} u, \stackrel{\bullet}{D} \pi_{\mathcal{B D}(D)} v\right) \in h$. This shows $(u, v) \in \mathcal{D}(A)$ and hence, $A$ is closed.

Now we are able to state the main result of this subsection.

Proposition 4.4. Let $h$ be maximal monotone. Then $A$ is maximal monotone, too.

Proof. Since every maximal monotone relation is closed (in fact it is even demi-closed, see Subsection 2.2), the operator $A$ is closed according to 4.3 . Moreover, by $4.2 A$ is monotone. Thus, by 2.1 , it suffices to prove that $(1+A)$ has a dense range. To this end let $f \in H_{\nu, 0}\left(\mathbb{R} ; \mathcal{D}\left(G_{c}\right)\right)$ and $g \in H_{\nu, 0}\left(\mathbb{R} ; \mathcal{D}\left(D_{c}\right)\right)$. 
We define ${ }^{7}$

$$
\begin{aligned}
\widetilde{u} & :=\left(1-D G_{c}\right)^{-1} f-D\left(1-G_{c} D\right)^{-1} g \in H_{\nu, 0}\left(\mathbb{R} ; \mathcal{D}\left(D G_{c}\right)\right), \\
\widetilde{v} & :=\left(1-G_{c} D\right)^{-1} g-G_{c}\left(1-D G_{c}\right)^{-1} f \in H_{\nu, 0}(\mathbb{R} ; \mathcal{D}(D)),
\end{aligned}
$$

as well as

$$
\begin{aligned}
& u:=\pi_{\mathcal{B D}(G)}^{*}(1+h)^{-1}\left(-\stackrel{\bullet}{D} \pi_{\mathcal{B D}(D)} G_{c} \widetilde{u}\right)+\widetilde{u} \in H_{\nu, 0}(\mathbb{R} ; \mathcal{D}(G)) \\
& v:=-\pi_{\mathcal{B D}(D)}^{*} \dot{G}(1+h)^{-1}\left(-\stackrel{\bullet}{D} \pi_{\mathcal{B D}(D)} G_{c} \widetilde{u}\right)+\widetilde{v} \in H_{\nu, 0}(\mathbb{R} ; \mathcal{D}(D)) .
\end{aligned}
$$

We show that $(u, v) \in \mathcal{D}(A)$ and that $(1+A)\left(\begin{array}{l}u \\ v\end{array}\right)=\left(\begin{array}{l}f \\ g\end{array}\right)$. First, we compute

$$
\begin{aligned}
\widetilde{u}+D \widetilde{v}= & \left(1-D G_{c}\right)^{-1} f-D\left(1-G_{c} D\right)^{-1} g \\
& +D\left(\left(1-G_{c} D\right)^{-1} g-G_{c}\left(1-D G_{c}\right)^{-1} f\right) \\
= & \left(1-D G_{c}\right)\left(1-D G_{c}\right)^{-1} f \\
= & f,
\end{aligned}
$$

and

$$
\begin{aligned}
\widetilde{v}+G_{c} \widetilde{u}= & \left(1-G_{c} D\right)^{-1} g-G_{c}\left(1-D G_{c}\right)^{-1} f \\
& +G_{c}\left(\left(1-D G_{c}\right)^{-1} f-D\left(1-G_{c} D\right)^{-1} g\right) \\
= & \left(1-G_{c} D\right)\left(1-G_{c} D\right)^{-1} g \\
= & g .
\end{aligned}
$$

Moreover, we get that

$$
\begin{aligned}
& u+D v=\pi_{\mathcal{B D}(G)}^{*}(1+h)^{-1}\left(-\stackrel{\bullet}{D} \pi_{\mathcal{B D}(D)} G_{c} \widetilde{u}\right)+\widetilde{u} \\
& +D\left(-\pi_{\mathcal{B D}(D)}^{*} \stackrel{\bullet}{G}(1+h)^{-1}\left(-\stackrel{\bullet}{D} \pi_{\mathcal{B D}(D)} G_{c} \widetilde{u}\right)+\widetilde{v}\right) \\
& =\pi_{\mathcal{B D}(G)}^{*}(1+h)^{-1}\left(-\stackrel{\bullet}{D} \pi_{\mathcal{B D}(D)} G_{c} \widetilde{u}\right)+\widetilde{u}
\end{aligned}
$$

\footnotetext{
${ }^{7}$ Note that $1-G_{c} D$ and $1-D G_{c}$ are boundedly invertible, since $-G_{c} D$ and $-D G_{c}$ are positive selfadjoint operators.
} 


$$
\begin{aligned}
& -\pi_{\mathcal{B D}(G)}^{*} \stackrel{\bullet}{D} \dot{G}(1+h)^{-1}\left(-\dot{D} \pi_{\mathcal{B D}(D)} G_{c} \widetilde{u}\right)+D \widetilde{v} \\
= & \widetilde{u}+D \widetilde{v}=f
\end{aligned}
$$

as well as

$$
\begin{aligned}
& v+G u=-\pi_{\mathcal{B D}(D)}^{*} \stackrel{\bullet}{G}(1+h)^{-1}\left(-\dot{D} \pi_{\mathcal{B D}(D)} G_{c} \widetilde{u}\right)+\widetilde{v} \\
& +G\left(\pi_{\mathcal{B D}(G)}^{*}(1+h)^{-1}\left(-\stackrel{\bullet}{D} \pi_{\mathcal{B D}(D)} G_{c} \widetilde{u}\right)+\widetilde{u}\right) \\
& =\widetilde{v}+G_{c} \widetilde{u}=g,
\end{aligned}
$$

which shows that $\left(1+\left(\begin{array}{cc}0 & D \\ G & 0\end{array}\right)\right)\left(\begin{array}{l}u \\ v\end{array}\right)=\left(\begin{array}{l}f \\ g\end{array}\right)$. Hence, the only thing which is left to show is $\left(\pi_{\mathcal{B D}(G)} u, \stackrel{\bullet}{D} \pi_{\mathcal{B D}(D)} v\right) \in h$. Since $\widetilde{u}$ takes values in $\mathcal{D}\left(G_{c}\right)$ we get that

$$
\pi_{\mathcal{B D}(G)} u=(1+h)^{-1}\left(-\stackrel{\bullet}{D} \pi_{\mathcal{B D}(D)} G_{c} \widetilde{u}\right)
$$

Using that

$$
\begin{aligned}
\pi_{\mathcal{B D}(D)} G_{c} D\left(1-G_{c} D\right)^{-1} g=-\pi_{\mathcal{B D}(D)} g+\pi_{\mathcal{B D}(D)}\left(1-G_{c} D\right)^{-1} g \\
=\pi_{\mathcal{B D}(D)}\left(1-G_{c} D\right)^{-1} g
\end{aligned}
$$

we obtain

$$
\begin{aligned}
\pi_{\mathcal{B D}(D)} G_{c} \widetilde{u} & =\pi_{\mathcal{B D}(D)} G_{c}\left(\left(1-D G_{c}\right)^{-1} f-D\left(1-G_{c} D\right)^{-1} g\right) \\
& =\pi_{\mathcal{B D}(D)} G_{c}\left(1-D G_{c}\right)^{-1} f-\pi_{\mathcal{B D}(D)}\left(1-G_{c} D\right)^{-1} g \\
& =-\pi_{\mathcal{B D}(D)} \widetilde{v} .
\end{aligned}
$$

Thus, we compute

$$
\begin{aligned}
\pi_{\mathcal{B D}(D)} v+\dot{G} \pi_{\mathcal{B D}(G)} u= & -\dot{G}(1+h)^{-1}\left(-\dot{D} \pi_{\mathcal{B D}(D)} G_{c} \widetilde{u}\right)+\pi_{\mathcal{B D}(D)} \widetilde{v} \\
& +\dot{G}(1+h)^{-1}\left(-\dot{D} \pi_{\mathcal{B D}(D)} G_{c} \widetilde{u}\right)
\end{aligned}
$$




$$
=\pi_{\mathcal{B D}(D)} \widetilde{v}=-\pi_{\mathcal{B D}(D)} G_{c} \widetilde{u},
$$

which gives

$$
\dot{D}_{\mathcal{B D}(D)} v+\pi_{\mathcal{B D}(G)} u=-\dot{D} \pi_{\mathcal{B D}(D)} G_{c} \widetilde{u} .
$$

Together with 8 the latter yields

$$
\pi_{\mathcal{B D}(G)} u=(1+h)^{-1}\left(\stackrel{\bullet}{D} \pi_{\mathcal{B D}(D)} v+\pi_{\mathcal{B D}(G)} u\right),
$$

which is equivalent to $\left(\pi_{\mathcal{B D}(G)} u, \stackrel{\bullet}{D} \pi_{\mathcal{B D}(D)} v\right) \in h$.

Summarizing we have shown that if $h$ satisfies the hypothesis (H3), then so does $A$, which follows by 4.2. Moreover, it follows that if $h$ satisfies (H2), then $A$ satisfies (H2) as well. Indeed, the maximal monotonicity was shown in 4.4 while the statement that $A$ is autonomous if $h$ is autonomous follows directly by the definition of $A$. Hence, we have proved 4.1.

\subsection{Applications}

In this subsection we apply our findings of the Sections 3 and 4.1 to two examples. The first problem is linear and deals with the model of acoustic waves with an impedance-type boundary condition (see [17]). The second one, describing a frictional contact problem for a visco-elastic medium, was also treated by Migorski et al. (see [9]) for an antiplane model, using a variational approach.

\section{Wave Propagation with Impedance-Type Boundary Conditions}

Throughout let $\Omega \subseteq \mathbb{R}^{n}$. We define the following differential operators, which will play the role of $G$ and $D$.

Definition. We define $\operatorname{grad}_{c}$ as the closure of the operator

$$
\begin{aligned}
\left.\operatorname{grad}\right|_{C_{c}^{\infty}(\Omega)}: C_{c}^{\infty}(\Omega) \subseteq L_{2}(\Omega) & \rightarrow L_{2}(\Omega)^{n} \\
\phi & \mapsto\left(\partial_{i} \phi\right)_{i \in\{1, \ldots, n\}}
\end{aligned}
$$

and likewise $\operatorname{div}_{c}$ as the closure of

$$
\begin{aligned}
\left.\operatorname{div}\right|_{C_{c}^{\infty}(\Omega)^{n}}: C_{c}^{\infty}(\Omega)^{n} \subseteq L_{2}(\Omega)^{n} & \rightarrow L_{2}(\Omega) \\
\left(\psi_{i}\right)_{i \in\{1, \ldots, n\}} & \mapsto \sum_{i=1}^{n} \partial_{i} \psi_{i} .
\end{aligned}
$$


Remark 4.5. The operators $\operatorname{grad}_{c}$ and $\operatorname{div}_{c}$ are formally skew-adjoint and as above we set grad $:=-\left(\operatorname{div}_{c}\right)^{*}$ and div $:=-\left(\operatorname{grad}_{c}\right)^{*}$. Note that an element $u$ lying in the domain of $\operatorname{grad}_{c}$ satisfies the abstract Dirichlet-boundary condition " $u=0$ " on $\partial \Omega$. Likewise, an element $q \in \mathcal{D}\left(\operatorname{div}_{c}\right)$ satisfies the Neumannboundary condition " $q \cdot N=0$ " on $\partial \Omega$ in case of a smooth boundary $\partial \Omega$, where $N$ denotes the unit outer normal vector-field on $\partial \Omega$. The corresponding boundary data spaces are given by $\mathcal{B D}(\operatorname{grad})=[\{0\}](1-$ div grad $)$, the space of 1-harmonic functions on $\Omega$, and $\mathcal{B D}($ div $)=[\{0\}](1-\operatorname{grad}$ div $)$.

Following [17] we consider the following boundary value problem:

$$
\begin{aligned}
\partial_{0, \nu}^{2} u-\operatorname{div} \operatorname{grad} u & =f \text { on } \Omega, \\
\left(\partial_{0, \nu}^{2} a\left(\partial_{0, \nu}^{-1}\right) u+\operatorname{grad} u\right) \cdot N & =0 \text { on } \partial \Omega .
\end{aligned}
$$

Here $a: B_{\mathbb{C}}(r, r) \rightarrow L\left(L_{2}(\Omega)^{n}\right)$ is a linear material law for some $r>0$, having the power series representation

$$
a(z)=\sum_{k=0}^{\infty} a_{k}(m)(z-r)^{k},
$$

where the $a_{k}$ 's are $L_{\infty}(\Omega)$-vector fields, and $a_{k}(m)$ denotes the corresponding multiplication operator. Moreover we assume that $\operatorname{div} a_{k} \in L_{\infty}(\Omega)$ such that

$$
(\operatorname{div} a): B_{\mathbb{C}}(r, r) \ni z \mapsto \sum_{k=0}^{\infty}\left(\operatorname{div} a_{k}\right)(m)(z-r)^{k} \in L\left(L_{2}(\Omega)\right)
$$

gets bounded. We choose $\nu>\frac{1}{2 r}$. Using the product rule $\operatorname{div}\left(a\left(\partial_{0, \nu}^{-1}\right) p\right)=$ $\left((\operatorname{div} a)\left(\partial_{0, \nu}^{-1}\right)\right) p+a\left(\partial_{0, \nu}^{-1}\right) \operatorname{grad} p$ for $p \in \mathcal{D}(\operatorname{grad})$, we can establish $a\left(\partial_{0, \nu}^{-1}\right)$ as a bounded operator

$$
a\left(\partial_{0, \nu}^{-1}\right): H_{\nu, 0}(\mathbb{R} ; \mathcal{D}(\operatorname{grad})) \rightarrow H_{\nu, 0}(\mathbb{R} ; \mathcal{D}(\operatorname{div})) .
$$

Moreover, one can show that $a\left(\partial_{0, \nu}^{-1}\right)\left[H_{\nu, 0}\left(\mathbb{R} ; \mathcal{D}\left(\operatorname{grad}_{c}\right)\right)\right] \subseteq H_{\nu, 0}\left(\mathbb{R} ; \mathcal{D}\left(\operatorname{div}_{c}\right)\right)$.

We define

$$
h: \mathcal{D}(h) \subseteq H_{\nu, 0}(\mathbb{R} ; \mathcal{B D}(\operatorname{grad})) \rightarrow H_{\nu, 0}(\mathbb{R} ; \mathcal{B D}(\operatorname{grad}))
$$

by $h:=\partial_{0, \nu} \operatorname{div} \pi_{\mathcal{B D}(\operatorname{div})} a\left(\partial_{0, \nu}^{-1}\right) \pi_{\mathcal{B} \mathcal{D}(\text { grad })}^{*}$ with maximal domain, which is a densely defined closed linear operator. Consider the operator

$$
A: \mathcal{D}(A) \subseteq H_{\nu, 0}\left(\mathbb{R} ; L_{2}(\Omega) \oplus L_{2}(\Omega)^{n}\right) \rightarrow H_{\nu, 0}\left(\mathbb{R} ; L_{2}(\Omega) \oplus L_{2}(\Omega)^{n}\right)
$$




$$
\left(\begin{array}{c}
u \\
q
\end{array}\right) \mapsto\left(\begin{array}{cc}
0 & \operatorname{div} \\
\operatorname{grad} & 0
\end{array}\right)\left(\begin{array}{l}
u \\
q
\end{array}\right)
$$

where

$$
\begin{aligned}
& \mathcal{D}(A) \\
& :=\left\{(u, q) \in H_{\nu, 0}(\mathbb{R} ; \mathcal{D}(\operatorname{grad}) \oplus \mathcal{D}(\operatorname{div})) \mid h\left(\pi_{\mathcal{B D}(\text { grad })} u\right)=\dot{\operatorname{div}} \pi_{\mathcal{B D}(\operatorname{div})} q\right\} .
\end{aligned}
$$

Note that, by definition, the operator $h$ is autonomous in the sense of (H2). The evolutionary equation for the boundary value problem 9,10 is then given by

$$
\left(\partial_{0, \nu}+A\right)\left(\begin{array}{c}
u \\
q
\end{array}\right)=\left(\begin{array}{c}
\partial_{0, \nu}^{-1} f \\
0
\end{array}\right)
$$

Indeed, if $\left(\begin{array}{c}u \\ q\end{array}\right)$ is a solution of 13 then we formally get that $\partial_{0, \nu} q+\operatorname{grad} u=0$, which gives $q=-\partial_{0, \nu}^{-1} \operatorname{grad} u$. Moreover, the first line of 13 gives $\partial_{0, \nu} u+\operatorname{div} q=$ $\partial_{0, \nu}^{-1} f$. Hence, the formula for $q$ yields $\partial_{0, \nu} u-\partial_{0, \nu}^{-1} \operatorname{div} \operatorname{grad} u=\partial_{0, \nu}^{-1} f$, which gives 9. Moreover, by the domain of $A$ we get that

$$
\begin{aligned}
\stackrel{\bullet}{\operatorname{div} \pi_{\mathcal{B D}(\text { div })} q} & =h\left(\pi_{\mathcal{B D}(\text { grad })} u\right) \\
& =\partial_{0, \nu} \stackrel{\bullet}{\operatorname{div}} \pi_{\mathcal{B D}(\operatorname{div})} a\left(\partial_{0, \nu}^{-1}\right) \pi_{\mathcal{B D}(\text { grad })}^{*} \pi_{\mathcal{B D}(\text { grad })} u \\
& =\partial_{0, \nu} \dot{\operatorname{div}} \pi_{\mathcal{B D}(\operatorname{div})} a\left(\partial_{0, \nu}^{-1}\right) u
\end{aligned}
$$

where in the third equality we have used that $a\left(\partial_{0, \nu}^{-1}\right)\left[H_{\nu, 0}\left(\mathbb{R} ; \mathcal{D}\left(\operatorname{grad}_{c}\right)\right)\right] \subseteq$ $H_{\nu, 0}\left(\mathbb{R} ; \mathcal{D}\left(\operatorname{div}_{c}\right)\right)$. Since $q=-\partial_{0, \nu}^{-1} \operatorname{grad} u$ the latter equation gives

$$
-\partial_{0, \nu}^{-2} \pi_{\mathcal{B D}(\text { div })} \operatorname{grad} u=\pi_{\mathcal{B D}(\text { div })} a\left(\partial_{0, \nu}^{-1}\right) u
$$

which is the appropriate formulation for the boundary condition 10 within our framework. According to our findings in Subsection 4.1 it suffices to check, whether $h$ satisfies the hypotheses (H2) and (H3). In [17] we find an additional constraint on $a\left(\partial_{0, \nu}^{-1}\right)$, namely

$$
\operatorname{Re} \int_{-\infty}^{0}\left(\left\langle\operatorname{grad} u \mid \partial_{0, \nu} a\left(\partial_{0, \nu}^{-1}\right) u\right\rangle(t)+\left\langle u \mid \operatorname{div} \partial_{0, \nu} a\left(\partial_{0, \nu}^{-1}\right) u\right\rangle(t)\right) e^{-2 \nu t} \mathrm{~d} t \geq 0
$$


for all $u \in H_{\nu, 0}(\mathbb{R} ; \mathcal{D}(\operatorname{grad})) \cap \mathcal{D}\left(\partial_{0, \nu}\right)$. This condition implies the hypothesis (H3). Indeed, for $u \in \mathcal{D}\left(\partial_{0, \nu}\right) \cap H_{\nu, 0}(\mathbb{R} ; \mathcal{B D}($ grad $))$ we estimate

$$
\begin{aligned}
& \operatorname{Re} \int_{-\infty}^{0}\langle h u \mid u\rangle_{\mathcal{B D}(\operatorname{grad})}(t) e^{-2 \nu t} \mathrm{~d} t \\
& =\operatorname{Re} \int_{-\infty}^{0}\left\langle\partial_{0, \nu} \operatorname{div} P_{\mathcal{B D}(\operatorname{div})} a\left(\partial_{0, \nu}^{-1}\right) \pi_{\mathcal{B D}(\operatorname{grad})}^{*} u \mid \pi_{\mathcal{B D}(\operatorname{grad})}^{*} u\right\rangle_{\mathcal{D}(\operatorname{grad})}(t) e^{-2 \nu t} \mathrm{~d} t \\
& =\operatorname{Re} \int_{-\infty}^{0}\left\langle P_{\mathcal{B D}(\operatorname{div})} \partial_{0, \nu} a\left(\partial_{0, \nu}^{-1}\right) \pi_{\mathcal{B D}(\operatorname{grad})}^{*} u \mid \operatorname{grad} \pi_{\mathcal{B D}(\operatorname{grad})}^{*} u\right\rangle(t) e^{-2 \nu t} \mathrm{~d} t \\
& +\operatorname{Re} \int_{-\infty}^{0}\left\langle\operatorname{div} P_{\mathcal{B D}(\operatorname{div})} \partial_{0, \nu} a\left(\partial_{0, \nu}^{-1}\right) \pi_{\mathcal{B D}(\text { grad })}^{*} u \mid \pi_{\mathcal{B D}(\text { grad })}^{*} u\right\rangle(t) e^{-2 \nu t} \mathrm{~d} t \\
& =\operatorname{Re} \int_{-\infty}^{0}\left\langle\partial_{0, \nu} a\left(\partial_{0, \nu}^{-1}\right) \pi_{\mathcal{B D}(\operatorname{grad})}^{*} u \mid \operatorname{grad} \pi_{\mathcal{B D}(\operatorname{grad})}^{*} u\right\rangle(t) e^{-2 \nu t} \mathrm{~d} t \\
& -\operatorname{Re} \int_{-\infty}^{0}\left\langle P_{\mathcal{D}\left(\operatorname{div}_{c}\right)} \partial_{0, \nu} a\left(\partial_{0, \nu}^{-1}\right) \pi_{\mathcal{B D}(\operatorname{grad})}^{*} u \mid \operatorname{grad} \pi_{\mathcal{B D}(\operatorname{grad})}^{*} u\right\rangle(t) e^{-2 \nu t} \mathrm{~d} t \\
& +\operatorname{Re} \int_{-\infty}^{0}\left\langle\operatorname{div} \partial_{0, \nu} a\left(\partial_{0, \nu}^{-1}\right) \pi_{\mathcal{B D}(\text { grad })}^{*} u \mid \pi_{\mathcal{B D}(\text { grad })}^{*} u\right\rangle(t) e^{-2 \nu t} \mathrm{~d} t \\
& -\operatorname{Re} \int_{-\infty}^{0}\left\langle\operatorname{div}_{c} P_{\mathcal{D}\left(\operatorname{div}_{c}\right)} \partial_{0, \nu} a\left(\partial_{0, \nu}^{-1}\right) \pi_{\mathcal{B D}(\operatorname{grad})}^{*} u \mid \pi_{\mathcal{B D}(\operatorname{grad})}^{*} u\right\rangle(t) e^{-2 \nu t} \mathrm{~d} t \\
& =\operatorname{Re} \int_{-\infty}^{0}\left\langle\partial_{0, \nu} a\left(\partial_{0, \nu}^{-1}\right) \pi_{\mathcal{B D}(\operatorname{grad})}^{*} u \mid \operatorname{grad} \pi_{\mathcal{B D}(\text { grad })}^{*} u\right\rangle(t) e^{-2 \nu t} \mathrm{~d} t \\
& +\operatorname{Re} \int_{-\infty}^{0}\left\langle\operatorname{div} \partial_{0, \nu} a\left(\partial_{0, \nu}^{-1}\right) \pi_{\mathcal{B D}(\operatorname{grad})}^{*} u \mid \pi_{\mathcal{B D}(\operatorname{grad})}^{*} u\right\rangle(t) e^{-2 \nu t} \mathrm{~d} t \\
& \geq 0 \text {. }
\end{aligned}
$$

Since $\mathcal{D}\left(\partial_{0, \nu}\right) \cap H_{\nu, 0}(\mathbb{R} ; \mathcal{B D}(\operatorname{grad}))$ is a core of $h$, according to 3.2 and since $h$ commutes with the operators $\tau_{h}$ for each $h \in \mathbb{R}$, this yields the monotonicity of 
$h$. For showing the maximal monotonicity of $h$, we determine its adjoint. Using 3.2 we get that

$$
h^{*}=\partial_{0, \nu}^{*} \pi_{\mathcal{B D}(\mathrm{grad})} a\left(\partial_{0, \nu}^{-1}\right)^{*} \pi_{\mathcal{B D}(\text { div })}^{*} \stackrel{\bullet}{\operatorname{grad}},
$$

and we obtain that $\mathcal{D}\left(\partial_{0, \nu}\right) \cap H_{\nu, 0}(\mathbb{R} ; \mathcal{B D}(\operatorname{grad}))=\mathcal{D}\left(\partial_{0, \nu}^{*}\right) \cap H_{\nu, 0}(\mathbb{R} ; \mathcal{B D}(\operatorname{grad}))$ is a core of $h^{*}$. Hence, $h^{*}$ is monotone, since for each

$$
u \in \mathcal{D}\left(\partial_{0, \nu}\right) \cap H_{\nu, 0}(\mathbb{R} ; \mathcal{B D}(\operatorname{grad})) \subseteq \mathcal{D}(h)
$$

we estimate

$$
\operatorname{Re}\left(h^{*} u|u\rangle_{H_{\nu, 0}(\mathbb{R} ; \mathcal{B D}(\mathrm{grad}))}=\operatorname{Re}|u| h u\right\rangle_{H_{\nu, 0}(\mathbb{R} ; \mathcal{B D}(\mathrm{grad}))} \geq 0 .
$$

We summarize our findings in the following theorem.

Theorem 4.6. Let $a: B_{\mathbb{C}}(r, r) \rightarrow L\left(L_{2}(\Omega)^{n}\right)$ be of the form 11 and let $a$ satisfy 12 and 14 . Then the evolutionary equation 13 is well-posed and the corresponding solution operator is causal.

Proof. Since $h$ and $h^{*}$ are monotone, we get the maximal monotonicity of $h$, since $h$ is closed. Thus, $h$ satisfies the hypotheses (H2) and (H3). 4.1 and 3.7 now give the well-posedness of 13 and 3.10 shows the causality of the solution operator.

\section{Visco-Elasticity with Frictional Boundary Conditions}

This subsection is devoted to the study of the following system:

$$
\begin{aligned}
\varrho \partial_{0, \nu}^{2} u-\operatorname{Div} T & =f \\
T & =C \operatorname{Grad} u+D \operatorname{Grad} \partial_{0, \nu} u
\end{aligned}
$$

on a domain $\Omega \subseteq \mathbb{R}^{n}$ completed by a nonlinear boundary condition of the form

$$
\left(\partial_{0, \nu} u,-T \cdot N\right) \in g,
$$

where $g \subseteq H_{\nu, 0}\left(\mathbb{R} ; L_{2}(\partial \Omega)\right) \oplus H_{\nu, 0}\left(\mathbb{R} ; L_{2}(\partial \Omega)\right)$ and $N$ denotes the outward unit normal vector-field on $\partial \Omega$. Here $u \in H_{\nu, 0}\left(\mathbb{R} ; L_{2}(\Omega)^{n}\right)$, denoting the displacementfield of the body $\Omega$, and $T \in H_{\nu, 0}\left(\mathbb{R} ; H_{\text {sym }}(\Omega)\right)$, standing for the stress tensor, are the unknowns. Equation 15 is the well-known equation of elasticity, describing the deformation of an elastic body $\Omega$ with a density distribution $\varrho \in L_{\infty}(\Omega)$, 
which is assumed to be real-valued and strictly positive. The operator Div denotes the divergence for symmetric-matrix-valued functions and the function $f \in H_{\nu, 0}\left(\mathbb{R} ; L_{2}(\Omega)^{n}\right)$ is an external force. The constitutive relation 16, linking the stress $T$ and the strain Grad $u$, where by Grad we denote the symmetrized Jacobian of a vector-valued function, is known as the Kelvin-Voigt model in visco-elasticity. Here $C$ and $D$ denote the elasticity and the viscosity tensor, respectively. The boundary condition 17 is motivated by so-called frictional boundary conditions, where $g$ is usually a subgradient of a convex, lower semicontinuous function (due to Rockafellar and Tyrell [22]) or a generalized gradient of a locally Lipschitz-continuous function (due to Clarke [3]). For typical examples of frictional boundary conditions in elasticity we refer to $[25$, p. 171 ff.] and to [9, Section 5].

A similar problem was treated in [9] for antiplane shear deformations, where the domain $\Omega$ was assumed to be a cylinder in $\mathbb{R}^{3}$. In this case the displacement can be expressed by a scalar-valued function and hence, 15 reduces to an equation similar to 9 . The boundary condition 17 was assumed to hold on a part of the boundary, while on the other parts of $\partial \Omega$ Dirichlet- and Neumann-boundary conditions were prescribed. The relation $g$ was assumed to be a generalized gradient of a locally Lipschitz-continuous function and the existence of a solution was proved, using a variational approach. The uniqueness of a solution was shown under the additional constraint that $g$ is quasi-monotone (i.e. there exists some constant $c>0$ such that $g+c$ is monotone). Here, for simplicity we assume that 17 holds on the whole boundary and that $g$ satisfies a suitable monotonicity constraint. However, since our relation is allowed to act in time and space, it covers a broader class of possible boundary conditions.

We begin by introducing the differential operators Grad and Div.

Definition. Let $\Omega \subseteq \mathbb{R}^{n}$ be open and denote by $L_{2}(\Omega)^{n \times n}$ the space of $n \times n$-matrix-valued $L_{2}(\Omega)$-functions equipped with the Frobenius inner product given by

$$
\langle\Phi \mid \Psi\rangle:=\int_{\Omega} \operatorname{trace}\left(\Phi(t)^{*} \Psi(t)\right) \mathrm{d} t \quad\left(\Phi, \Psi \in L_{2}(\Omega)^{n \times n}\right) .
$$

Moreover, we set $H_{\text {sym }}(\Omega):=\left\{\Phi \in L_{2}(\Omega)^{n \times n} \mid \Phi(x)^{T}=\Phi(x) \quad(x \in \mathbb{R}\right.$ a.e. $\left.)\right\}$, which is a closed subspace of $L_{2}(\Omega)^{n \times n}$ and hence, a Hilbert space. We define the operator $\operatorname{grad}_{c}$ as the closure of

$$
\begin{aligned}
\left.\operatorname{grad}\right|_{C_{c}^{\infty}(\Omega)^{n}}: C_{c}^{\infty}(\Omega)^{n} \subseteq L_{2}(\Omega)^{n} & \rightarrow L_{2}(\Omega)^{n \times n} \\
\left(\phi_{i}\right)_{i \in\{1, \ldots, n\}} & \mapsto\left(\partial_{j} \phi_{i}\right)_{i, j \in\{1, \ldots, n\}}
\end{aligned}
$$


and $\operatorname{div}_{c}$ as the closure of

$$
\begin{aligned}
\left.\operatorname{div}\right|_{C_{c}^{\infty}(\Omega)^{n \times n}: C_{c}^{\infty}(\Omega)^{n \times n} \subseteq L_{2}(\Omega)^{n \times n}} \rightarrow L_{2}(\Omega)^{n} \\
\left(\Psi_{i j}\right)_{i, j \in\{1, \ldots, n\}} \mapsto\left(\sum_{j=1}^{n} \partial_{j} \Psi_{i j}\right)_{i \in\{1, \ldots, n\}}
\end{aligned}
$$

Furthermore, we introduce the operators $\operatorname{Grad}_{c}$ and $\operatorname{Div}_{c}$ as the closures of

$$
\begin{aligned}
\left.\operatorname{Grad}\right|_{C_{c}^{\infty}(\Omega)^{n}: C_{c}^{\infty}(\Omega)^{n} \subseteq L_{2}(\Omega)^{n}} \rightarrow H_{\mathrm{sym}}(\Omega) \\
\left(\phi_{i}\right)_{i \in\{1, \ldots, n\}} \mapsto\left(\frac{1}{2}\left(\partial_{i} \phi_{j}+\partial_{j} \phi_{i}\right)\right)_{i, j \in\{1, \ldots, n\}}
\end{aligned}
$$

and of

$$
\begin{aligned}
\left.\operatorname{Div}\right|_{C_{c}^{\infty}(\Omega)^{n \times n} \cap H_{\mathrm{sym}}(\Omega)}: C_{c}^{\infty}(\Omega)^{n \times n} \cap H_{\mathrm{sym}}(\Omega) \subseteq & H_{\mathrm{sym}}(\Omega) \rightarrow L_{2}(\Omega)^{n} \\
\left(\Psi_{i j}\right)_{i, j \in\{1, \ldots, n\}} & \mapsto\left(\sum_{j=1}^{n} \partial_{j} \Psi_{i j}\right)_{i \in\{1, \ldots, n\}},
\end{aligned}
$$

respectively ${ }^{8}$.

Remark 4.7. An easy computation shows that $\operatorname{grad}_{c}$ and $\operatorname{div}_{c}$ as well as $\operatorname{Grad}_{c}$ and $\operatorname{Div}_{c}$ are formally skew-adjoint and we define grad $:=\left(-\operatorname{div}_{c}\right)^{*} \supseteq$ $\operatorname{grad}_{c}, \operatorname{div}:=\left(-\operatorname{grad}_{c}\right)^{*} \supseteq \operatorname{div}_{c}$ as well as Grad $:=-\left(\operatorname{Div}_{c}\right)^{*} \supseteq \operatorname{Grad}_{c}$ and $\operatorname{Div}:=-\left(\operatorname{Grad}_{c}\right)^{*} \supseteq \operatorname{Div}_{c}$.

Throughout we may assume that $\mathcal{D}(\mathrm{Grad}) \stackrel{\iota}{\hookrightarrow} \mathcal{D}(\mathrm{grad})$, i.e. Korn's inequality holds (for sufficient conditions for Korn's inequality we refer to [2] and the references therein). We rewrite the equations 15 and 16 into a system of the form 4. We assume that $D \in L\left(H_{\text {sym }}(\Omega)\right)$ is a selfadjoint, strictly positive definite operator and hence, we may rewrite 16 as follows

$$
T=\left(C+D \partial_{0, \nu}\right) \operatorname{Grad} u=\partial_{0, \nu} D\left(\partial_{0, \nu}^{-1} D^{-1} C+1\right) \operatorname{Grad} u,
$$

which gives

$$
\partial_{0, \nu}^{-1} D^{-1} T=\left(\partial_{0, \nu}^{-1} D^{-1} C+1\right) \operatorname{Grad} u
$$

\footnotetext{
${ }^{8}$ By definition, elements of $\mathcal{D}\left(\operatorname{Grad}_{c}\right)$ and of $\mathcal{D}\left(\operatorname{Div}_{c}\right)$ satisfy a generalized boundary condition of the form $\phi=0$ on $\partial \Omega$ and $\Psi \cdot N=0$ on $\partial \Omega$ for $\phi \in \mathcal{D}\left(\operatorname{Grad}_{c}\right)$ and $\Psi \in \mathcal{D}\left(\operatorname{Div}_{c}\right)$, respectively, where $N$ denotes the outward normal vector-field on $\partial \Omega$.
} 
If we choose $\nu>0$ large enough, such that $\left\|D^{-1} C\right\|<\nu$, the latter equation can be written as

$$
\partial_{0, \nu}^{-1}\left(\partial_{0, \nu}^{-1} D^{-1} C+1\right)^{-1} D^{-1} T=\operatorname{Grad} u
$$

Thus, the equations 15 and 16 can be replaced by the system

$$
\left(\partial_{0, \nu}\left(\begin{array}{lc}
\varrho & 0 \\
0 & \partial_{0, \nu}^{-1}\left(\partial_{0, \nu}^{-1} D^{-1} C+1\right)^{-1} \\
D^{-1}
\end{array}\right)+\left(\begin{array}{cc}
0 & \operatorname{Div} \\
\operatorname{Grad} & 0
\end{array}\right)\right)\left(\begin{array}{c}
v \\
-T
\end{array}\right)=\left(\begin{array}{l}
f \\
0
\end{array}\right)
$$

where $v:=\partial_{0, \nu} u$. Hence, the material law $M\left(\partial_{0, \nu}^{-1}\right)$ is given by

$$
M\left(\partial_{0, \nu}^{-1}\right)=\left(\begin{array}{ll}
\varrho & 0 \\
0 & 0
\end{array}\right)+\partial_{0, \nu}^{-1}\left(\begin{array}{cc}
0 & 0 \\
0 & D^{-1}
\end{array}\right)-\partial_{0, \nu}^{-2}\left(\begin{array}{cc}
0 & 0 \\
0 & \left(\partial_{0, \nu}^{-1} C+D\right)^{-1} C D^{-1}
\end{array}\right) .
$$

According to the assumptions on $\varrho$ and $D$, this material law satisfies the hypothesis (H1) if we choose $\nu>0$ large enough (this corresponds to the so-called 0 -analytic case in [15]).

We now have to formulate the boundary condition 17 in our framework. Since we do not want to impose regularity assumptions on the boundary $\partial \Omega$, we have to detour the space $L_{2}(\partial \Omega)$. Following [19, p. $16 \mathrm{ff}$.] we define the following substitute for $L_{2}(\partial \Omega)$.

Definition. We assume that $N \in L_{\infty}(\Omega)^{n}$ with $\operatorname{div} N \in L_{\infty}(\Omega) .{ }^{9} \mathrm{We}$ define the operator $\nu: \mathcal{B D}($ grad $) \rightarrow \mathcal{B D}($ div $)$ by $\nu f:=\pi_{\mathcal{B D}(\text { div })}\left(f_{j} N_{k}\right)_{j, k \in\{1, \ldots, n\}}$ and assume that

$$
\left\langle\left(\stackrel{\bullet}{\operatorname{div} \nu+\nu^{*} \operatorname{grad}}\right) f \mid f\right\rangle_{\mathcal{B D}(\operatorname{grad})}>0 \quad(f \in \mathcal{B D}(\operatorname{grad}) \backslash\{0\}) .
$$

This gives that $(f, g) \mapsto \frac{1}{2}\left(\langle\nu f \mid \stackrel{\bullet \operatorname{grad} g}{ }\rangle_{\mathcal{B D}(\text { div })}+\langle\underset{\operatorname{grad} f \mid}{\bullet} \mid \nu\rangle_{\mathcal{B D}(\text { div })}\right)^{\text {de- }}$ fines an inner product on $\mathcal{B D}(\mathrm{grad})$ and we denote the completion of $\mathcal{B D}$ (grad) with respect to this inner product by $U$. Moreover, we denote the embedding of $\mathcal{B D}($ grad $)$ into $U$ by $\kappa$.

\footnotetext{
${ }^{9}$ This could be interpreted as a "smooth" continuation of the outward normal vector field $N$ to the whole domain $\Omega$.
} 
Remark 4.8. A formal calculation (see [19, Remark 5.2]) yields that in the case of a smooth boundary we have that

$$
\langle f \mid g\rangle_{U}=\int_{\partial \Omega} f(x)^{*} g(x) \mathrm{d} S(x) .
$$

We define the mapping $j:=\kappa \pi_{\mathcal{B D}(\operatorname{grad})} \iota \pi_{\mathcal{B D}(\mathrm{Grad})}^{*}: \mathcal{B D}(\mathrm{Grad}) \rightarrow U$. Then, as it was pointed out in [19, Remark 5.4], the term $\operatorname{Grad} j^{*} u$ can be identified with $u$ on $\partial \Omega$. This construction allows us to compare Dirichlet- and Neumann-type boundary values.

Using this framework, we assume that $g$ is a binary relation on $H_{\nu, 0}(\mathbb{R} ; U)$. Then the boundary condition 17 can be formulated as

$$
\left(\pi_{\mathcal{B D}(\text { Grad })} v, \pi_{\mathcal{B D}(\text { Div })}(-T)\right) \in \stackrel{\bullet}{\operatorname{Grad}} j^{*} g j
$$

or equivalently as

$$
\left(\pi_{\mathcal{B D}(\mathrm{Grad})} v, \stackrel{\bullet}{\operatorname{Div}} \pi_{\mathcal{B D}(\text { Div })}(-T)\right) \in j^{*} g j .
$$

We define the operator $A$ by

$$
\begin{gathered}
\mathcal{D}(A):=\left\{(v, \sigma) \in H_{\nu, 0}(\mathbb{R} ; \mathcal{D}(\mathrm{Grad}) \oplus \mathcal{D}(\text { Div })) \mid\right. \\
\qquad\left(\begin{array}{c}
v \\
\left.\left.\pi_{\mathcal{B D}(\mathrm{Grad})} v, \stackrel{\operatorname{Div}}{ } \pi_{\mathcal{B D}(\text { Div })} \sigma\right) \in j^{*} g j\right\} \\
\sigma
\end{array}\right):=\left(\begin{array}{cc}
0 & \text { Div } \\
\operatorname{Grad} & 0
\end{array}\right)\left(\begin{array}{c}
v \\
\sigma
\end{array}\right),
\end{gathered}
$$

and consider the equation

$$
\left(\partial_{0, \nu}\left(\begin{array}{lc}
\varrho & 0 \\
0 & \partial_{0, \nu}^{-1}\left(\partial_{0, \nu}^{-1} D^{-1} C+1\right)^{-1} D^{-1}
\end{array}\right)+A\right)\left(\begin{array}{l}
v \\
\sigma
\end{array}\right)=\left(\begin{array}{c}
f \\
0
\end{array}\right),
$$

which is, according to our findings, an appropriate reformulation of the problem given by 15, 16 and 17. Hence, we are in the situation of Subsection 4.1 and if we assume that $j^{*} g j \subseteq H_{\nu, 0}(\mathbb{R} ; \mathcal{B D}(\mathrm{Grad})) \oplus H_{\nu, 0}(\mathbb{R} ; \mathcal{B D}(\mathrm{Grad}))$ satisfies the hypotheses (H2) and (H3), 4.1 applies, which gives the well-posedness and the causality of the problem. We summarize our findings in the following theorem. 
Theorem 4.9. Let $\nu>\left\|D^{-1} C\right\|$ be such that $M\left(\partial_{0, \nu}^{-1}\right)$, given by 18 , satisfies (H1) and let $g \subseteq H_{\nu, 0}(\mathbb{R} ; U) \oplus H_{\nu, 0}(\mathbb{R} ; U)$ be such that

$$
j^{*} g j \subseteq H_{\nu, 0}(\mathbb{R} ; \mathcal{B D}(\mathrm{Grad})) \oplus H_{\nu, 0}(\mathbb{R} ; \mathcal{B D}(\mathrm{Grad}))
$$

satisfies the hypotheses (H2) and (H3). Then the problem given by 19, where $A$ is defined by 4.2 is well-posed and the corresponding solution operator is causal.

In the last part of this subsection we will present a condition on $g$, which implies the maximal monotonicity of $j^{*} g j$.

In [9] the relation $g$ is defined as the canonical extension (see 3.1 (a)) of a quasi-monotone relation $\widetilde{g}$ on $L_{2}(\partial \Omega)$, which is given by

$$
\widetilde{g}:=\left\{(u, v) \in L_{2}(\partial \Omega) \oplus L_{2}(\partial \Omega) \mid(u(x), v(x)) \in \partial j(x, \cdot) \quad(x \in \partial \Omega \text { a.e. })\right\},
$$

where $j(\cdot, \cdot): \partial \Omega \times \mathbb{R} \rightarrow \mathbb{R}$ is a suitable function, which is locally Lipschitzcontinuous with respect to the second variable. Moreover it is assumed that $[\mathbb{R}] \partial j(x, \cdot)=\mathbb{R}$ for almost every $x \in \partial \Omega$ and that there exists $c>0$ such that for every $\xi \in \mathbb{R}$ and every $\eta \in \mathbb{R}$ satisfying $(\xi, \eta) \in \partial j(x, \cdot)$ the estimate $|\eta| \leq c(1+|\xi|)$ holds. This gives that $\left[L_{2}(\partial \Omega)\right] \widetilde{g}=L_{2}(\partial \Omega)$ and that $\widetilde{g}$ is a bounded relation, i.e. $\widetilde{g}[M]$ is bounded for bounded $M$.

A suitable realization of these assumptions in our framework is given as follows. We assume that $\widetilde{g} \subseteq U \oplus U$ is a maximal monotone bounded relation, satisfying $[U] \widetilde{g}=U$ and for technical reasons $(0,0) \in \widetilde{g}$. In the next lemma we show that these conditions imply that $j^{*} \widetilde{g} j$ is maximal monotone, too. This would give that $j^{*} g j$ satisfies the hypotheses (H2) and (H3), where $g \subseteq H_{\nu, 0}(\mathbb{R} ; U) \oplus H_{\nu, 0}(\mathbb{R} ; U)$ is the canonical extension of $\widetilde{g}$.

Lemma 4.10. Let $H_{1}, H_{2}$ be two Hilbert spaces, $T \subseteq H_{1} \oplus H_{1}$ maximal monotone and bounded with $(0,0) \in T$ and $\left[H_{1}\right] T=H_{1}$. Moreover, let $S \in$ $L\left(H_{1}, H_{2}\right)$. Then $S T S^{*} \subseteq H_{2} \oplus H_{2}$ is maximal monotone.

Proof. The monotonicity of $S T S^{*}$ is clear. We will show that

$$
\left(1+S T S^{*}\right)\left[H_{2}\right]=H_{2}
$$

which implies the maximal monotonicity according to 2.1. Let $f \in H_{2}$. We replace the relation $T$ by its Yosida-approximation $T_{\lambda}$, which is monotone and Lipschitz-continuous with a Lipschitz-constant less than or equal to $\lambda^{-1}$. Hence, $S T_{\lambda} S^{*}$ is monotone and Lipschitz-continuous with a Lipschitz-constant less than or equal to $\frac{\|S\|^{2}}{\lambda}$. For $\mu<\frac{\lambda}{\|S\|^{2}}$ the contraction mapping theorem yields the 
existence of an element $x \in H_{2}$ satisfying $x+\mu S T_{\lambda}\left(S^{*} x\right)=y$. Hence, the mapping $S T_{\lambda} S^{*}$ is maximal monotone and thus, for each $\lambda>0$ there exists $x_{\lambda} \in$ $H_{2}$ with $x_{\lambda}+S T_{\lambda}\left(S^{*} x_{\lambda}\right)=y$. The latter can be written as $x_{\lambda}=J_{1}\left(S T_{\lambda} S^{*}\right)(y)$, which implies that $\left|x_{\lambda}\right| \leq|y|$ for each $\lambda>0$, according to 2.2 (a). Since $T$ is bounded, this gives that $\left\{T_{\lambda}\left(S^{*} x_{\lambda}\right) \mid \lambda>0\right\}$ is bounded (see $2.2(\mathrm{~b})$ ). We will prove that $\left(x_{\lambda}\right)_{\lambda>0}$ converges as $\lambda$ tends to 0 . For $\mu, \lambda>0$ we estimate

$$
\begin{aligned}
\left|x_{\lambda}-x_{\mu}\right|^{2}= & \operatorname{Re}\left(x_{\lambda}-x_{\mu}\left|S T_{\mu}\left(S^{*} x_{\mu}\right)-S T_{\lambda}\left(S^{*} x_{\lambda}\right)\right\rangle\right. \\
= & \operatorname{Re}\left(S^{*} x_{\lambda}-S^{*} x_{\mu}\left|T_{\mu}\left(S^{*} x_{\mu}\right)-T_{\lambda}\left(S^{*} x_{\lambda}\right)\right\rangle\right. \\
= & \operatorname{Re}\left(\lambda T_{\lambda}\left(S^{*} x_{\lambda}\right)-\mu T_{\mu}\left(S^{*} x_{\mu}\right)\left|T_{\mu}\left(S^{*} x_{\mu}\right)-T_{\lambda}\left(S^{*} x_{\lambda}\right)\right\rangle\right. \\
& +\operatorname{Re}\left(J_{\lambda}(T)\left(S^{*} x_{\lambda}\right)-J_{\mu}(T)\left(S^{*} x_{\mu}\right)\left|T_{\mu}\left(S^{*} x_{\mu}\right)-T_{\lambda}\left(S^{*} x_{\lambda}\right)\right\rangle\right. \\
\leq & \operatorname{Re}\left(\lambda T_{\lambda}\left(S^{*} x_{\lambda}\right)-\mu T_{\mu}\left(S^{*} x_{\mu}\right)\left|T_{\mu}\left(S^{*} x_{\mu}\right)-T_{\lambda}\left(S^{*} x_{\lambda}\right)\right\rangle\right. \\
\leq & (\lambda+\mu) C,
\end{aligned}
$$

for a suitable constant $C>0$. This gives that $\left(x_{\lambda}\right)_{\lambda>0}$ converges to some $x \in H_{1}$ as $\lambda \rightarrow 0$. Moreover, $\left|J_{\lambda}(T)\left(S^{*} x_{\lambda}\right)-S^{*} x\right| \leq\left|J_{\lambda}(T)\left(S^{*} x_{\lambda}\right)-S^{*} x_{\lambda}\right|+$ $\left|S^{*} x_{\lambda}-S^{*} x\right|=\lambda\left|T_{\lambda}\left(S^{*} x_{\lambda}\right)\right|+\left|S^{*} x_{\lambda}-S^{*} x\right| \rightarrow 0$ as $\lambda \rightarrow 0$. Furthermore, since $\left\{T_{\lambda}\left(S^{*} x_{\lambda}\right) \mid \lambda>0\right\}$ is bounded there exists a weakly convergent subsequence $\left(T_{\lambda_{n}}\left(S^{*} x_{\lambda_{n}}\right)\right)_{n \in \mathbb{N}}$ with $\lambda_{n} \rightarrow 0$ as $n \rightarrow \infty$ and we denote its weak limit by $z \in H_{1}$. Then, since $\left(J_{\lambda}(T)\left(S^{*} x_{\lambda}\right), T_{\lambda}\left(S^{*} x_{\lambda}\right)\right) \in T$ for each $\lambda>0$, the demiclosedness of $T$ implies $\left(S^{*} x, z\right) \in T$. Since

$$
S T_{\lambda}\left(S^{*} x_{\lambda}\right)=y-x_{\lambda} \rightarrow y-x,
$$

we have that $S z=y-x$, which gives $(x, y-x) \in S T S^{*}$ or equivalently $(x, y) \in$ $1+S T S^{*}$.

\section{Acknowledgments}

The author thanks Marcus Waurick for careful reading and fruitful discussions and the editor for his kind support.

\section{References}

[1] H. Brezis, Operateurs maximaux monotones et semi-groupes de contractions dans les espaces de Hilbert, Universite Paris VI et CNRS, 1971. 
[2] P. G. Ciarlet and P. jun. Ciarlet, Another approach to linearized elasticity and a new proof of Korn's inequality, Math. Models Methods Appl. Sci., 15(2):259-271, 2005.

[3] F. H. Clarke and W. U. M. M. R. Center, Generalized Gradients of Lipschitz Functionals, Defense Technical Information Center, 1976.

[4] P. Do Panagiotopoulos, Inequality Problems in Mechanic and Applications: Convex and Nonconvex Energy Functions, Birkhäuser, 1985.

[5] S. Hu and N. S. Papageorgiou, Handbook of Multivalued Analysis, volume 1: Theory, Springer, 1997.

[6] S. Hu and N. S. Papageorgiou, Handbook of Multivalued Analysis, volume 2: Applications, of Mathematics and its applications, Kluwer Academic Publishers, 2000.

[7] A. Kalauch, R. Picard, S. Siegmund, S. Trostorff, and M. Waurick, A Hilbert Space Perspective on Ordinary Differential Equations with Memory Term, Technical report, TU Dresden, 2011, arXiv:1204.2924.

[8] V. Lakshmikantham, S. Leela, and F. A. Mcrae, Theory of Causal Differential Equations, Atlantis Studies in Mathematics for Engineering and Science. World Scientific Pub Co Inc, 2010.

[9] S. Migórski, A. Ochal, and M. Sofonea, Solvability of dynamic antiplane frictional contact problems for viscoelastic cylinders, Nonlinear Analysis: Theory, Methods \& Applications, 70(10):3738-3748, 2009.

[10] S. Migórski, A. Ochal, and M. Sofonea, Analysis of a dynamic contact problem for electro-viscoelastic cylinders, Nonlinear Analysis: Theory, Methods \& Applications, 73(5):1221-1238, 2010.

[11] S. Migórski, A. Ochal, and M. Sofonea, A dynamic frictional contact problem for piezoelectric materials, Journal of Mathematical Analysis and Applications, 361(1):161-176, 2010.

[12] G. Minty, Monotone (nonlinear) operators in a hilbert space, Duke Math. J., 29, 1962.

[13] G. Morosanu, Nonlinear evolution equations and applications, Springer, 2nd edition, 1988. 
[14] Z. Naniewicz, On some nonmonotone subdifferential boundary conditions in elastostatics, Ingenieur-Archiv, 60:31-40, 1989.

[15] R. Picard, A structural observation for linear material laws in classical mathematical physics, Math. Methods Appl. Sci., 32(14):1768-1803, 2009.

[16] R. Picard, On a comprehensive class of linear material laws in classical mathematical physics, Discrete Contin. Dyn. Syst., Ser. S, 3(2):339-349, 2010.

[17] R. Picard, A class of evolutionary problems with an application to acoustic waves with impedance type boundary conditions, In Spectral Theory, Mathematical System Theory, Evolution Equations, Differential and Difference Equations, volume 221 of Operator Theory: Advances and Applications, pages 533-548. Springer Basel, 2012.

[18] R. Picard and D. McGhee, Partial differential equations. A unified Hilbert space approach, de Gruyter Expositions in Mathematics 55. Berlin: de Gruyter. xviii, 2011.

[19] R. Picard, S. Trostorff, and M. Waurick, On a Class of Boundary Control Systems, Technical report, TU Dresden, 2012, arXiv:1211.3634.

[20] R. Picard, S. Trostorff, and M. Waurick, On a comprehensive Class of Linear Control Problems, Technical report, TU Dresden, 2012, arXiv:1208.3140.

[21] R. H. Picard, Hilbert space approach to some classical transforms, Pitman research notes in mathematics series. Longman Scientific \& Technical, 1989.

[22] R. Rockafellar, Convex Analysis, Princeton, N. J.: Princeton University Press, XVIII, 1970.

[23] W. Rudin, Real and complex analysis, Mathematics series. McGraw-Hill, 1987.

[24] R. E. Showalter, Monotone Operators in Banach Space and Nonlinear Partial Differential Equations, American Mathematical Society, 1997.

[25] M. Sofonea and A. Matei, Variational inequalities with applications. A study of antiplane frictional contact problems, Advances in Mechanics and Mathematics 18. New York, NY: Springer. xix, 2009. 
[26] S. Trostorff, Well-posedness and causality for a class of evolutionary inclusions, PhD thesis, TU Dresden, 2011, URL: http://www.qucosa.de/fileadmin/data/qucosa/documents/7832/phdthesis_trostorff.pdf.

[27] S. Trostorff, An alternative approach to well-posedness of a class of differential inclusions in Hilbert spaces, Nonlinear Anal., Theory Methods Appl., Ser. A, Theory Methods, 75(15):5851-5865, 2012.

[28] S. Trostorff, Well-posedness of Linear Integro-Differential Equations with Operator-valued Kernels, Technical report, TU Dresden, 2012, arXiv:1210.1728. 